\title{
Model Predictive Control for Electrodynamic Tether Geometric Profile in Orbital Maneuvering with Finite Element State Estimator
}

\section{Gangqiang Li}

York University - Keele Campus: York University

Zheng H. Zhu ( $\sim$ gzhu@yorku.ca )

York University https://orcid.org/0000-0002-0149-0473

\section{Research Article}

Keywords: Model predictive control, State estimation, Finite element method, Kalman filter, Underactuated, Electrodynamic tether

Posted Date: May 13th, 2021

DOl: https://doi.org/10.21203/rs.3.rs-474272/v1

License: (c) (i) This work is licensed under a Creative Commons Attribution 4.0 International License.

Read Full License 


\title{
Model Predictive Control for Electrodynamic Tether Geometric Profile in Orbital Maneuvering with Finite Element State Estimator
}

\author{
Gangqiang Li, Zheng H. Zhu* \\ Department of Mechanical Engineering, York University \\ 4700 Keele Street, Toronto, Ontario, Canada M3J 1P3
}

\begin{abstract}
:
This paper studies the control of geometric profile of a librating electrodynamic tether by model predictive control using the induced electric current in tether only. First, a high-fidelity multiphysics model of an electrodynamic tether system is built based on the nodal position finite element method and the orbital-motion-limited theory. Second, a state estimator is proposed to estimate the geometric profile of a librating electrodynamic tether, where only the positions and velocities at the tether ends are measurable. The non-measurable geometric profile of tether between two ends is estimated by the high-fidelity multiphysics model with the input of the measurement at tether ends in the spatial domain. To avoid the singularity or ambiguity in the estimation, the geometric profile of tether is then propagated in the time domain by the extended Kalman filter. Third, the problem of controlling the geometric profile of a librating electrodynamic tether is converted into a trajectory tracking problem of the underactuated electrodynamic tether system, where the induced electric current in the tether is the only control input. The control input is optimized by the model predictive control method subject to the output and input control constraints. The numerical simulation results show that the proposed approach is capable of effectively controlling the shape of the liberating electrodynamic tether to the reference trajectory.

*Corresponding author. Tel.: +1 4167362100 x 77729, Fax: +1 416736 5817. E-mail address: gzhu@ yorku.ca.
\end{abstract}


Keywords: Model predictive control; State estimation; Finite element method; Kalman filter; Underactuated; Electrodynamic tether.

\section{Introduction}

Space tethers, especially the bare Electrodynamic tethers (EDT), have been proposed for removing space debris from Earth orbits for decades because of low mass, compact size, propellant-free, and ease of operation [1-4]. However, the application of the EDT is impeded by its intrinsically unstable libration motion [2], which is caused by the continuous interaction of the current-carrying EDT with the Earth magnetic field [3,5]. Many efforts have been devoted to stabilizing the libration motions of an EDT by regulating the electric current in tether as the only control input [5-8]. The tether was first modeled by a simple dumbbell model to apply analytical control laws $[8,9]$ with constant electric current assumption [10]. Later, a flexible tether model was proposed to discretize the tether into a finite number of segments with an energy-rate feedback control law [7]. Here, the geometric profile of the tether is assumed measurable by Kalman filter. Unfortunately, it is practically impossible to place sensors along the thin tether to measure its spatial position. Nevertheless, the discretized tether model results in multiple sets of (in-plane and out-of-plane) libration angles, see Fig. 1, instead of only one set of libration angles in the dumbbell model. This renders the existing libration control strategies based on the dumbbell model. To mitigate this problem, the authors proposed a virtual straight tether that connects the first and last notes of the flexible tether model to represent the libration motion, see Fig. 1, and developed a libration stabilization strategy by electric current on-off control scheme to keep the Hamiltonian of the virtual tether bounded [5, 11]. Although effective, this approach works only if the real tether is approximately straight. Once the EDT is bent by orbital perturbative forces, the Hamiltonian of 
the virtual tether no longer represents the real EDT, and the energy-based libration control scheme fails [11].

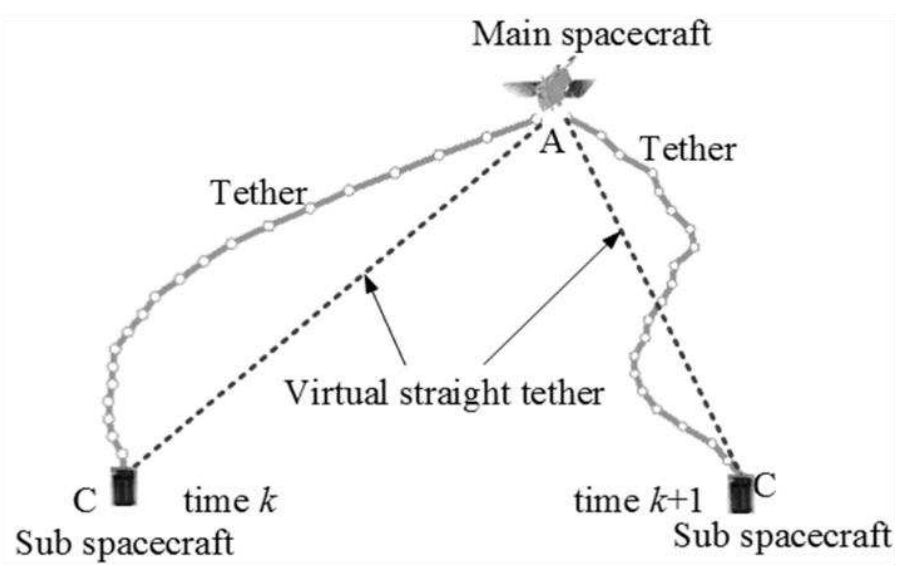

Fig. 1. Schematic diagram of a discretized EDT with virtual straight tethers.

Motivated by the challenge, this work develops a finite element model-based approach that is able to estimate the geometric profile of tether by input of positions and velocities at the tether ends only and then to keep it straight. First, the spatially continuous dynamical system is discretized into finite elements (FE). The FE model establishes the relationship in the spatial domain between the measured state at the tether ends of and the non-measurable state along the tether. Then, the extended Kalman filter is applied to propagate the full state (measured and non-measurable) in the time domain to avoid the singularity in case the degrees of freedom of non-measurable state are greater than that of measured state. Random noises are introduced in the measurement model to account for the sensor noises, unmodeled dynamics and model uncertainties associated with the finite element method [12]. Once the full state estimation is obtained, the model predictive control (MPC) method is applied to control the geometric profile of EDT by minimizing the difference between the real and virtual straight tethers as shown in Fig. 1. 


\section{High-fidelity dynamic model of electrodynamic tether}

\subsection{System description}

Consider an EDT orbiting the Earth as shown in Fig. 2. It consists of a main and a sub spacecraft connected by an elastic tether that is bare and electrically conductive [5]. Electric potential bias is induced in the tether as the latter interacts with the Earth magnetic field. Electrons in ambient plasma are attracted by positively charged segment of the bare EDT $[2,13]$ and emitted back to the plasma by an emitter at the cathodic end [13], resulting in an electric current in the EDT. The current-carrying EDT interacts with the Earth magnetic field and results in a Lorentz (electrodynamic) force against EDT's orbit motion.

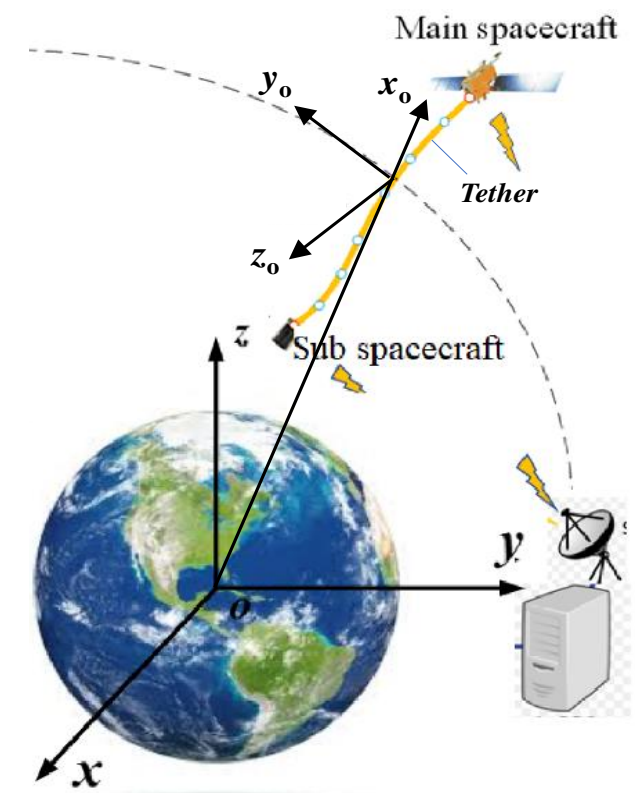

(a)

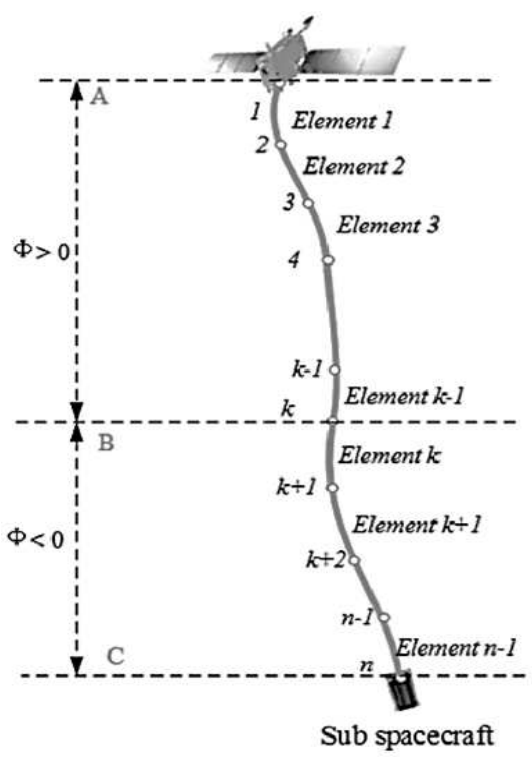

(b)

Fig. 2. The schematic diagram of a flexible EDT model.

Four orthogonal coordinate systems are defined to describe the motion of an EDT [11, 14] as shown in Fig. 2. The first is the global inertial coordinate system (oxyz) with its origin $\boldsymbol{o}$ at the Earth center, $\boldsymbol{x}$-axis in the vernal equinox, $\boldsymbol{z}$-axis aligned with the Earth's rotation axis, and $\boldsymbol{y}$-axis 
forming a right-hand coordinate system. The second is the Earth-fixed coordinate system ( $\boldsymbol{o} \boldsymbol{x}_{f} \boldsymbol{y}_{f} \boldsymbol{z}_{f}$ ) where the gravity field, atmosphere and ionospheric plasmas densities of the Earth are described [14]. The $\boldsymbol{o} \boldsymbol{x}_{f} \boldsymbol{y}_{f} \boldsymbol{z}_{f}$ is assumed to coincide with the $\boldsymbol{o x y z}$ initially. The third is the orbital coordinate system $\left(\boldsymbol{o}_{o} \boldsymbol{x}_{o} \boldsymbol{y}_{o} \boldsymbol{z}_{o}\right)$ with the origin of $\boldsymbol{o}_{o} \boldsymbol{x}_{o} \boldsymbol{y}_{o} \boldsymbol{z}_{o}$ at the center of mass (CM) of the EDT system, $\boldsymbol{x}_{\boldsymbol{o}}$-axis from the center of the Earth to the CM, $\boldsymbol{y}_{\boldsymbol{o}}$-axis in the orbital motion direction, and $z_{\boldsymbol{o}}$-axis forming a right-hand system. Finally, the local coordinate system $\left(\boldsymbol{o}_{l} \boldsymbol{t n} \boldsymbol{b}\right)$ is defined at the element level. The origin $\boldsymbol{o}_{l}$ is at element's CM and the detailed definition can be found in [11, $14,15]$.

\subsection{High-fidelity dynamic model of EDT}

The EDT is modeled by a high-fidelity multiphysics model based on the nodal position finite element method $[16,17]$. To make the paper self-contented for interested readers, this model is briefly described here. The main and sub spacecraft are simplified as lumped masses due to the extremely large ratio of the tether length over the size of the spacecraft. Next, the tether is discretized into (n-1) two-noded elastic tensile elements with $n$ nodes. The dynamic model of the discretized EDT is derived by the principle of virtual work $[11,17]$,

$$
\boldsymbol{m}(t)+\boldsymbol{c}(t) \mathfrak{d}(t)+\boldsymbol{k}(t) \mathrm{x}(t)=\boldsymbol{f}_{g}(t)+\boldsymbol{f}_{d}(t)+\boldsymbol{f}_{l}(t)
$$

where the over dot denotes time derivative, $\boldsymbol{x}(t) \in \mathbf{R}^{3 n}$ is the vector of nodal position, ( $\boldsymbol{m}$ and $\left.\boldsymbol{k}(t) \in i^{3 n \times 3 n}\right)$ are the mass and stiffness matrices, and $\boldsymbol{c}(t)=\alpha_{1} \boldsymbol{m}+\alpha_{2} \boldsymbol{k}(t)$ is the Rayleigh damping with $\alpha_{1}$ and $\alpha_{2}$ being damping coefficients $[12,16]$. It is noted the mass matrix $\boldsymbol{m}$ is a constant matrix, which is advantageous in numerical integration $[16,17]$. The lumped masses of spacecraft are added directly to the diagonal elements mass matrix $\boldsymbol{m}$ corresponding to the first 
and last nodes. The terms $\left(\boldsymbol{f}_{g}(t), \boldsymbol{f}_{d}(t)\right.$ and $\left.\boldsymbol{f}_{l}(t) \in \mathrm{i}^{3 n}\right)$ are the gravity, atmosphere drag, and electrodynamic force, respectively.

The gravity and atmosphere drag are evaluated by,

$$
\begin{gathered}
\boldsymbol{f}_{g}(t)=\sum_{j=1}^{n-1}\left(\int_{0}^{l_{e, j}} \boldsymbol{S}^{T} \rho_{j} \boldsymbol{g}_{j}^{g}(t) a_{j}(t) d l\right) \\
\boldsymbol{f}_{d}(t)=\sum_{j=1}^{n-1}\left(\int_{0}^{l_{e, j}} \boldsymbol{S}^{T} \boldsymbol{T}^{f 2 g}(t) \boldsymbol{T}^{l 2 f}(t) \boldsymbol{f}_{d, j}^{l}(t) d l\right) \\
\boldsymbol{f}_{d, j}^{l}(t)=\left(0, f_{d, j}^{l, n}, 0\right)^{T} ; \quad f_{d, j}^{l, n}=-\frac{1}{2} \rho_{a}(t) c_{d} d(t)\left|\not \mathbf{x}^{n}(t)-v_{a, j}^{l, n}(t)\right|\left[\operatorname{x女}_{j}^{n}(t)-v_{a, j}^{l, n}(t)\right]
\end{gathered}
$$

where the subscript $j \in[1, n-1]$ denotes the $j^{\text {th }}$ element, $\rho$ and $a$ are the material density and crosssection area of the tether, $l_{e, j}$ is the current length of the $j^{\text {th }}$ element, $\boldsymbol{S}$ is the matrix of linear shape function [17], $\boldsymbol{g}^{g}(t)=\boldsymbol{T}^{f 2 g}(t) \boldsymbol{g}^{f}(t)$ is the vector of gravity acceleration evaluated by the Earth's gravitational model [11] with the superscripts $g$ and $f$ representing in the global inertial and Earthfixed coordinate systems, $\boldsymbol{f}_{d}^{l}$ is the vector of atmospheric drag per unit length with the superscript $l$ representing the local frame, $\boldsymbol{T}^{f 2 g}(t)$ and $\boldsymbol{T}^{l 2 f}(t)$ represent the transformation: $\boldsymbol{o x} \boldsymbol{x}_{f} \boldsymbol{z}_{f}$ a $\boldsymbol{o x y z}$ and $\boldsymbol{o} l \boldsymbol{t n \boldsymbol { b }}$ a $\boldsymbol{o x} \boldsymbol{x}_{f} \boldsymbol{z}_{f}[5,11]$ respectively, $\rho_{a}$ is the atmosphere density evaluated by the Naval Research Laboratory Mass Spectrometer Incoherent Scatter Radar (NRLMSIS-00) model [14], $c_{d}$ is the drag coefficient $\left(c_{d}=2.2\right.$ for tether, $c_{d}=2.0$ for spacecraft $), d$ is the tether diameter, $\&$ and $v_{a}^{l}(t)=\left(v_{a}^{l, t}, v_{a}^{l, n}, v_{a}^{l, b}\right)^{T}$ are the velocity vectors of the EDT and surrounding atmosphere in the local coordinate system [17]. 
The electrodynamic force is evaluated by,

$$
\begin{aligned}
& \boldsymbol{f}_{l}(t)=\sum_{j=1}^{n-1}\left(\int_{0}^{l_{e, j}} \boldsymbol{S}^{T}\left\{\boldsymbol{T}^{l 2 g}(t)\left[-I_{j}(t)\left(\boldsymbol{b}_{j}^{l}(t) \times \boldsymbol{t}\right)\right]\right\} d l\right)=\boldsymbol{d}(t) \boldsymbol{u}(t) \\
& \boldsymbol{d}(t)=\boldsymbol{R}(t) \boldsymbol{G} \\
& \boldsymbol{R}=\operatorname{Diag}\left[\boldsymbol{T}_{1}^{l 2 g}(t), \mathrm{L}, \boldsymbol{T}_{m}^{l 2 g}(t)\right] \\
& \boldsymbol{G}=\left[\begin{array}{ccccc}
0.5 \boldsymbol{f}_{l, 1}^{l} l_{e, 1} & \boldsymbol{0} & \boldsymbol{0} & \mathrm{L} & \boldsymbol{0} \\
\boldsymbol{0} & 0.5\left(\boldsymbol{f}_{l, 1}^{l} l_{e, 1}+\boldsymbol{f}_{l, 2}^{l} l_{e, 2}\right) & \boldsymbol{0} & \mathrm{L} & \boldsymbol{0} \\
\boldsymbol{0} & \boldsymbol{0} & 0.5\left(\boldsymbol{f}_{L, 2}^{l} l_{e, 2}+\boldsymbol{f}_{L, 3}^{l} l_{e, 3}\right) & \mathrm{L} & \boldsymbol{0} \\
\boldsymbol{0} & \boldsymbol{0} & \boldsymbol{0} & \mathrm{O} & \mathrm{M} \\
\boldsymbol{0} & \boldsymbol{0} & \boldsymbol{0} & \mathrm{L} & 0.5 \boldsymbol{f}_{L, m}^{l} l_{e, m}
\end{array}\right] \\
& \boldsymbol{u}(t)=\left[\begin{array}{lllll}
I_{1}(t) & I_{2}(t) & I_{3}(t) & \mathrm{L} & I_{m}(t)
\end{array}\right]
\end{aligned}
$$

where $I_{j}(t)$ is the induced electric current in the $j^{\text {th }}$ element and assumed constant within element, $\boldsymbol{b}_{j}^{l}(t)$ is the magnetic field strength evaluated by the International Geomagnetic Reference Field model (2014) $[11,14]$ at the element CM, $t$ is a unit vector along the element axis in the local coordinate $[11,14], \boldsymbol{d}(t) \in \boldsymbol{i}^{3 n \times m}$ is the mapping matrix and $\boldsymbol{u}(t) \in \boldsymbol{i}^{m}$.

The bare EDT can be divided into the positively biased segment $A B$ that collects electrons and the negatively biased segment $B C$ that collects ions $[4,13]$ as shown in Fig. 2(b). The induced electric current $I(s, t)$ and potential bias $\Phi(s, t)$ obey the orbital motion limited (OML) theory $[4,13,18]$

$$
\left\{\begin{array}{l}
\frac{d I(s, t)}{d s}=\alpha_{1}(s, t) \alpha_{2}(s, t) \sqrt{\Phi(s, t)} \\
\frac{d \Phi(s, t)}{d s}=\alpha_{3}(s, t) I(s, t)-e_{m}(s, t)
\end{array} \Phi(s, t) \geq 0\right.
$$




$$
\left\{\begin{array}{l}
\frac{d I(s, t)}{d s}=-\frac{1}{172} \alpha_{1}(s, t) \alpha_{2}(s, t) \sqrt{-\Phi(s, t)} \\
\frac{d \Phi(s, t)}{d s}=\alpha_{3}(s, t) I(s, t)-e_{m}(s, t)
\end{array} \Phi(s, t)<0\right.
$$

where $s$ is the arc-length measured from the anodic end (point A), $\alpha_{i}(s, t)(i=1,2,3)$ are the coefficients relate to the electron density of ambient plasma and tether physical properties [4, 18], and $e_{m}(s, t)$ is the electric motive force (EMF) [14].

The boundary conditions that reflect the design of EDT system are chosen for the maximum efficiency of current generation for deorbit purpose [18], such that,

$$
\begin{array}{lll}
s=0, & I(0, t)=0, & \Phi(0, t)=\Phi_{A} \\
s=l_{B}(s, t), & I\left(l_{B}, t\right)=I_{B}, & \Phi\left(l_{B}, t\right)=\Phi_{B}=0 \\
s=l_{C}, & I\left(l_{C}, t\right)=I_{C}, & \Phi\left(l_{C}, t\right)=\Phi_{C}
\end{array}
$$

where $l_{B}$ is the length of positively biased segment of the tether and is unknown in priori [18], $l_{C}$ is the total length of EDT, $\Phi_{C}=\left|\Phi_{p w}\right|-\left|\Phi_{c h e}\right|+I_{C} Z_{t}$ is determined in priori by the gate potential bias $\Phi_{p w}$, the potential bias of battery $\Phi_{c h e}$, and the variable resistance $Z_{t}[4,18]$.

Equations (10)-(12) form a nonlinear two-point boundary value problem. They are discretized by FE method by the same mesh as in Eq. (1) $[4,18]$. If the point $B$ is coincided with the $p p^{\text {th }}$ node $(p p=2 \sim n-1)$, see Fig. 2(b), the discretized form of OML theory is written as

$$
\begin{gathered}
\boldsymbol{h}_{p o s, j} \boldsymbol{r}_{e d t, j}=\boldsymbol{f}_{p o s, j}^{e m f}(j=1, \mathrm{~K}, p p-1) \\
\boldsymbol{h}_{n e g, j} \boldsymbol{r}_{e d t, j}=\boldsymbol{f}_{n e g, j}^{e m f}(j=p p, \mathrm{~K}, n-1)
\end{gathered}
$$

where subscripts pos and neg denote the positive and negative element potential bias properties, $\boldsymbol{h}$ is the coefficient matrix, $\boldsymbol{r}_{\text {edt }}$ is the vector of nodal electric current and potential bias, and $\boldsymbol{f}_{\text {pos }}^{e m f}$ 
and $\boldsymbol{f}_{\text {neg }}^{e m f}$ are the corresponding EMF vectors [18].

Correspondingly, the boundary condition (12) becomes

$$
\begin{gathered}
\boldsymbol{h}_{1} \boldsymbol{r}_{e d t, 1}=0 \\
\boldsymbol{h}_{n} \boldsymbol{r}_{\text {edt }, n}=\left|\Phi_{p w}\right|-\left|\Phi_{c h e}\right|
\end{gathered}
$$

where $\boldsymbol{h}_{1}=\left(\begin{array}{llll}1 & 0 & 0 & 0\end{array}\right)$ and $\boldsymbol{h}_{n}=\left(\begin{array}{llll}0 & 0 & -Z_{T} & 1\end{array}\right)$.

If the point $B$ is inside an element, its location is first approximated by the solution of OML theory with the boundary conditions at two ends of element. Then, the element is divided into two subelements and modeled by Eqs. (13)-(14).

Finally, the discretized OML theory of an EDT system is assembled by a standard FE procedure

$$
\begin{aligned}
& \boldsymbol{z}=\boldsymbol{h}_{\text {edt }} \boldsymbol{r}_{\text {edt }}-\boldsymbol{f}^{e m f}=\boldsymbol{0} \\
& \boldsymbol{h}_{\text {edt }}=\left(\begin{array}{llllll}
\boldsymbol{h}_{1} & & & & & \\
\boldsymbol{h}_{p o s, 1} & & & & & \\
& \mathrm{O} & & & & \\
& & \boldsymbol{h}_{p o s, p p-1} & & & \\
& & & \boldsymbol{h}_{\text {neg, }, p p} & & \\
& & & \mathrm{O} & \\
& & & & \boldsymbol{h}_{\text {neg, }, n} \\
& & & & \boldsymbol{h}_{n}
\end{array}\right) \\
& \boldsymbol{r}_{e d t}=\left(\boldsymbol{r}_{e d t, 1} \mathrm{~L} \boldsymbol{r}_{e d t, p p-1}, \boldsymbol{r}_{e d t, p p}, \mathrm{~L} \boldsymbol{r}_{e d t, n-1}\right)^{T} \\
& \boldsymbol{f}^{e m f}=\left(\boldsymbol{0}, \boldsymbol{f}_{\text {pos }, 1}^{e m f}, \mathrm{~L}, \boldsymbol{f}_{p o s, p p-1}^{e m f}, \boldsymbol{f}_{\text {neg, }, p p}^{e m f}, \mathrm{~L}, \boldsymbol{f}_{\text {neg, }, n-1}^{e m f},\left|\Phi_{p w}\right|-\left|\Phi_{c h e}\right|\right)^{T}
\end{aligned}
$$


where $\boldsymbol{h}_{\text {edt }} \in \mathrm{i}^{2 n \times 2 n}, \boldsymbol{r}_{\text {edt }}=\left(I_{1}, \Phi_{1}, \mathrm{~K} I_{p p}, \Phi_{p p}, \mathrm{~K}, I_{n}, \Phi_{n}\right)^{T} \in i^{2 n}$ and $\boldsymbol{f}^{e m f} \in i^{2 n}[18]$

Equation (17) is a nonlinear equation and must be solved by the Newton-Raphson algorithm iteratively $[3,4,18]$ to obtain the electric current along the tether at each node, see Fig. 2(b)

$$
\boldsymbol{I}_{E D T}(t)=\left(I_{1}, \mathrm{~K}, I_{p p-1}, I_{p p}, \mathrm{~K}, I_{n}\right)^{T}
$$

where $\boldsymbol{I}_{E D T} \in \mathrm{i}^{n}$.

Recast Eq. (1) in the state-space form for MPC development,

$$
\begin{aligned}
& \boldsymbol{X}^{\ell}(t)=\boldsymbol{A}^{c}(t) \boldsymbol{X}(t)+\boldsymbol{B}^{c}(t) \boldsymbol{u}(t)+\boldsymbol{F}(t) \\
& \boldsymbol{X}(t)=\left[\begin{array}{c}
\boldsymbol{x}(t) \\
\boldsymbol{x}(t)
\end{array}\right], \quad \boldsymbol{A}^{c}(t)=\left[\begin{array}{cc}
\boldsymbol{0} & \boldsymbol{E}_{1} \\
-\boldsymbol{m}^{-1} \boldsymbol{k}(t) & -\boldsymbol{m}^{-1} c(t)
\end{array}\right] \\
& \boldsymbol{B}^{c}(t)=\left[\begin{array}{l}
\boldsymbol{0} \\
\boldsymbol{m}^{-1} \boldsymbol{d}(t)
\end{array}\right], \quad \boldsymbol{F}(t)=\left[\begin{array}{l}
\boldsymbol{0} \\
\boldsymbol{m}^{-1}\left(\boldsymbol{f}_{g}(t)+\boldsymbol{f}_{d}(t)\right)
\end{array}\right]
\end{aligned}
$$

where the superscript $c$ denotes the continuous time system, $\boldsymbol{E}_{1} \in \mathrm{i}^{3 n \times 3 n}$ is an identity matrix, and the control input (electric current) $\boldsymbol{u}(t) \in \mathfrak{i}^{m}$ must satisfy the following inequality,

$$
\boldsymbol{u}^{\text {low }}(t) \leq \boldsymbol{u}(t) \leq \boldsymbol{u}^{\text {upp }}(t)
$$

where $\boldsymbol{u}^{\text {low }}(t)$ and $\boldsymbol{u}^{\text {upp }}(t) \in \mathfrak{i}^{m}$ are the lower and upper bounds of control. For the deorbit application, $\boldsymbol{u}^{\text {low }}(t)=0$ and $\boldsymbol{u}^{\text {upp }}(t)=\boldsymbol{G} \boldsymbol{I}_{E D T}$ with $\boldsymbol{G} \in i^{m \times n}$ given in (9). As demonstrated in [3, 18], the value of $\boldsymbol{u}^{u p p}(t)$ depends on the libration motion, the geometric profile, and physical properties of EDT. Thus, $\boldsymbol{u}^{u p p}(t)$ is time variable and dependent on orbital parameters. 


\section{Model predictive control for geometric profile of electrodynamic tether}

\subsection{State estimator}

The full state of the tether is divided into two parts: the measured state at two ends of the EDT and the non-measurable state inside the tether. In the following, we will derive a state estimator like a virtual sensor to estimate the non-measurable state inside the tether, based on the input of measured state at the ends (boundary) of the tether in the temporal and spatial domains.

First, linearize the kinematics of the EDT system in the state-space as the system model,

$$
\begin{gathered}
\boldsymbol{X}_{o b s, k}=\boldsymbol{A}_{o b s, k-1}^{d} \boldsymbol{X}_{o b s, k-1}+\boldsymbol{B}_{o b s, k-1}^{d} \boldsymbol{u}_{k-1}+\boldsymbol{W}_{o b s, k-1} \\
\boldsymbol{A}_{o b s, k-1}^{d}=\left(\begin{array}{cc}
\boldsymbol{E}_{1} & \Delta t \boldsymbol{E}_{1} \\
\boldsymbol{0} & \boldsymbol{E}_{1}
\end{array}\right), \text { and } \boldsymbol{B}_{o b s, k-1}^{d}=\boldsymbol{0}
\end{gathered}
$$

where the superscript $d$ denotes the discrete time system, the subscript $o b s$ and $(k-1$ and $k)$ denotes the system model at the times $k$ and $k-1$, respectively, $\Delta t$ is the sampling interval, $\boldsymbol{W}_{o b s, k-1}$ is the noise accounting for the model and parameter uncertainties, $\boldsymbol{N}\left(\boldsymbol{0}, \boldsymbol{Q}_{\text {obs }}\right)$ represents the normal distribution with the zero mean $\boldsymbol{O}$ and covariance matrix $\boldsymbol{Q}_{o b s} \in \mathbf{R}^{6 n \times 6 n}$, and $\boldsymbol{u}_{k-1} \in \boldsymbol{i}^{m}$ is the vector of input control at time $k-1$.

Then, the measurement model is constructed in two parts: the measurable state at the boundary of the tether and the non-measurable state inside the tether.

For the measurable state $\boldsymbol{Y}_{o b s}^{1} \in i^{q_{1}=12}$ at the boundary of the tether $\partial \Omega$, the corresponding measurement model is defined as 


$$
\boldsymbol{Y}_{o b s, k-1}^{1}=\boldsymbol{H}_{o b s}^{1} \boldsymbol{X}_{o b s, k-1}+\boldsymbol{V}_{o b s, k-1}^{1}
$$

where the superscript 1 denotes the measurement model of measurable state, $\boldsymbol{H}_{o b s}^{1} \in \boldsymbol{i}^{q_{1} \times 6 n}$ is the measurement matrix and $\boldsymbol{V}_{o b s, k-1}^{1} \in i^{q_{1}}$ is the measurement noise of the sensors, such that,

$$
\boldsymbol{H}_{o b s}^{1}=\left[\begin{array}{cc}
\boldsymbol{h}_{\boldsymbol{1}} & \boldsymbol{0} \\
\boldsymbol{0} & \boldsymbol{h}_{1}
\end{array}\right] \text { and } \boldsymbol{h}_{\boldsymbol{1}}=\left[\begin{array}{ccc}
\boldsymbol{E}_{2} & \boldsymbol{0} \boldsymbol{\mathrm { O }} \boldsymbol{\boldsymbol { O }} & \boldsymbol{0} \\
\boldsymbol{0} & \boldsymbol{0} \mathrm{L} \boldsymbol{0} & \boldsymbol{E}_{2} / 2
\end{array}\right]
$$

Here, $\boldsymbol{E}_{2} \in i^{3 \times 3}$ is an identity matrix.

For the non-measurable state $\left(\boldsymbol{X}_{o b s}^{2} \in \mathbf{R}^{3 n-q_{1}}\right) \in \Omega$ inside the tether, the corresponding measurement model is derived from the finite element model of the EDT $[11,17]$ in the spatial domain with the input of measured state $\boldsymbol{Y}_{o b s}^{1} \in i^{q_{1}=12}$ at the boundary $\partial \Omega$, such that,

$$
\begin{gathered}
\boldsymbol{Y}_{o b s, k-1}^{2}=\boldsymbol{h}_{2}\left(\boldsymbol{X}_{o b s, k-1}\right)+\boldsymbol{V}_{o b s, k-1}^{2} \\
\boldsymbol{h}_{2}\left(\boldsymbol{X}_{o b s, k-1}\right)=\left\{\begin{array}{l}
\boldsymbol{m}_{o b s, k-1}+\boldsymbol{c}_{k t-1} \boldsymbol{H}_{o b s, k-1}+\boldsymbol{k}_{k-1} \boldsymbol{x}_{o b s, k-1}+ \\
\left(\frac{\partial \boldsymbol{G}_{k-1}}{\partial \boldsymbol{x}_{o b s}}\right)^{T} \lambda_{2}=\boldsymbol{f}_{g, k-1}+\boldsymbol{f}_{d, k-1}+\boldsymbol{f}_{l, k-1} \\
\boldsymbol{G}_{k-1}=\boldsymbol{Y}_{o b s, k-1}^{1}-\boldsymbol{H}_{o b s}^{1} \boldsymbol{X}_{o b s, k-1}-\boldsymbol{V}_{o b s, k-1}^{1}=\boldsymbol{O}
\end{array}\right.
\end{gathered}
$$

where the superscript 2 denotes the measurement model of non-measurable state, $\boldsymbol{Y}_{o b s, k-1}^{2} \in \mathbf{R}^{6 n-q_{1}}$ is the estimated state, $\boldsymbol{h}_{2}\left(\boldsymbol{X}_{o b s, k-1}\right) \in \mathbf{R}^{6 n-q_{1}}$ is an nonlinear virtual measurement function based on the spatially discretized finite element model of the flexible EDT, and $\boldsymbol{V}_{o b s, k-1}^{2} \in \mathbf{R}^{6 n-q_{1}}$ is the noise model that includes the noise propagated from the input of measured state at the boundary, the discretizing error of finite element method and unmodeled dynamics of the EDT. 
The $\boldsymbol{V}_{o b s, k-1}^{1}$ and $\boldsymbol{V}_{o b s, k-1}^{2}$ are assumed to be independent zero-mean Gaussian white-noise process, such that,

$$
\begin{aligned}
& \boldsymbol{V}_{o b s, k-1}^{1}: \boldsymbol{N}\left(\boldsymbol{0}, \boldsymbol{R}_{o b s}^{1}\right) \text { and } \boldsymbol{V}_{o b s, k-1}^{2}: \boldsymbol{N}\left(\boldsymbol{0}, \boldsymbol{R}_{o b s}^{2}\right) \\
& E X\left[\boldsymbol{V}_{o b s, k-1}^{1},{ }_{j} \boldsymbol{V}_{o b s, k-1}^{1}\right]=\boldsymbol{R}_{o b s}^{1} \delta_{i, j} \\
& E X\left[{ }_{i} \boldsymbol{V}_{o b s, k-1}^{2},{ }_{j} \boldsymbol{V}_{o b s, k-1}^{2}\right]=\boldsymbol{R}_{o b s}^{2} \delta_{i, j} \\
& E X\left[{ }_{i} \boldsymbol{V}_{o b s, k-1}^{1},{ }_{j} \boldsymbol{V}_{o b s, k-1}^{2}\right]=\mathbf{0}
\end{aligned}
$$

where $\delta_{i, j}$ and $E X[\cdot, \cdot]$ are the Kronecker delta and expectation functions, respectively, the subscripts $i$ and $j$ represents the $i$ and $j$ component of a vector, $\boldsymbol{R}_{o b s}^{1} \in \mathbf{R}^{q_{1} \times q_{1}}$ and $\boldsymbol{R}_{o b s}^{2} \in \mathbf{R}^{\left(6 n-q_{1}\right) \times\left(6 n-q_{1}\right)}$ are the known noise covariance matrices.

Equation (30) is numerically solved by the Lagrangian multiplier method directly [12]. However, the solution $\boldsymbol{Y}_{o b s}^{2}$ is not unique if $\left(6 n-q_{1}\right)>q_{1}$, i.e., the dimension of the non-measurable state vector $\boldsymbol{Y}_{o b s}^{2} \in \Omega$ is greater than that of the measurable state vector $\boldsymbol{Y}_{o b s}^{1}$ at the boundary $\partial \Omega$. To avoid the ambiguity or singularity in the reconstruction of the non-measurable internal state, the extended Kalman filter is used to propagate the full state of the EDT $\boldsymbol{X}_{k}$ with the known initial condition and the hybrid measurement model $\boldsymbol{Y}_{o b s}=\left(\begin{array}{ll}\boldsymbol{Y}_{o b s}^{1} & \boldsymbol{Y}_{o b s}^{2}\end{array}\right)^{T} \in \mathbf{R}^{6 n}$. This state estimator is defined as the finite element Kalman filter (FEKF) estimator that contains the prediction and correction stages [19-21] as follows.

\section{- Prediction step:}

$$
\begin{gathered}
\boldsymbol{X}_{o b s, k}^{-}=\boldsymbol{A}_{o b s, k-1}^{d} \boldsymbol{X}_{o b s, k-1}^{+} \\
\boldsymbol{P}_{o b s, k}^{-}=\boldsymbol{A}_{o b s, k-1}^{d} \boldsymbol{P}_{o b s, k-1}^{+}\left(\boldsymbol{A}_{o b s, k-1}^{d}\right)^{T}+\boldsymbol{Q}_{o b s}
\end{gathered}
$$




$$
\boldsymbol{K}_{o b s, k}=\boldsymbol{P}_{o b s, k}^{-} \boldsymbol{H}_{k}^{T}\left(\boldsymbol{H}_{k} \boldsymbol{P}_{o b s, k}^{-} \boldsymbol{H}_{k}^{T}+\boldsymbol{R}_{o b s}\right)^{-1}
$$

- Correction step:

$$
\begin{gathered}
\boldsymbol{X}_{o b s, k}^{+}=\boldsymbol{X}_{o b s, k}^{-}+\boldsymbol{K}_{o b s, k}\left[\boldsymbol{Y}_{o b s, k}-\boldsymbol{h}\left(\boldsymbol{X}_{o b s, k}^{-}\right)\right] \\
\boldsymbol{P}_{o b s, k}^{+}=\left(\boldsymbol{E}_{3}-\boldsymbol{K}_{o b s, k} \boldsymbol{H}_{k}^{+}\right) \boldsymbol{P}_{o b s, k}^{-}
\end{gathered}
$$

where the superscripts - or + denote prior or posterior estimators, $\boldsymbol{K}_{o b s}$ is the Kalman gain matrix, $\boldsymbol{h}\left(\boldsymbol{X}_{o b s, k}^{-}\right)=\left(\boldsymbol{H}^{1} \boldsymbol{X}_{o b s, k}^{-}, \boldsymbol{h}_{2}\left(\boldsymbol{X}_{o b s, k}^{-}\right)\right)^{T}, \boldsymbol{H}_{k}=\operatorname{Diag}\left(\boldsymbol{H}^{1}, \boldsymbol{H}_{k}^{2}\right)$ is the Jacobian matrix of the nonlinear measurement model with $\boldsymbol{H}_{k}^{2}=\partial \boldsymbol{h}_{2} /\left.\partial \boldsymbol{X}_{o b s}\right|_{X_{o b s}=X_{o b s, k}^{-}}$being the Jacobian matrix of the nonlinear virtual measurement function $\boldsymbol{h}_{2}(), \boldsymbol{R}_{o b s}=\operatorname{diag}\left(\boldsymbol{R}_{o b s}^{1}, \boldsymbol{R}_{o b s}^{2}\right)$, and $\boldsymbol{E}_{3} \in i^{6 n \times 6 n}$ is an identity matrix.

The stability of the newly proposed FEKF estimator can be proved based on the direct method of Lyapunov for the extended Kalman filter. The detailed proof can be found in [19, 20]. The observability of the newly proposed FEKF estimator is proved by the Popov-Belevitch-Hautus (PBH) test, see the page 174 of [22], and is briefly outlined here. The pair $\left(\boldsymbol{A}_{o b s}^{c}, \boldsymbol{H}\right)$ in the continuous system equation (22) and the measurement model is observable only and only if when the PBH matrix is a full column rank for all eigenvalues $\lambda \in \mathbb{R}$, such that,

$$
P B H=\left[\begin{array}{c}
\lambda \boldsymbol{E}_{1}-\boldsymbol{A}_{o b s}^{c} \\
\boldsymbol{H}
\end{array}\right]
$$

where $\boldsymbol{A}_{o b s}^{c}=\operatorname{diag}\left(\boldsymbol{E}_{1}, \boldsymbol{0}\right)$ is the state transition matrix with the superscript $c$ representing the continuous time system. 
Since the term $\left(\lambda \boldsymbol{E}_{1}-\boldsymbol{A}_{\text {obs }}^{c}\right)$ becomes singular only at the eigenvalues of the observed system, it suffices to merely investigate the observability of the system modes [22, 23]. The measurement matrix $\boldsymbol{H}_{k t}=\operatorname{diag}\left(\boldsymbol{H}^{1}, \boldsymbol{H}_{k t}^{2}\right)$ is known, and only the diagonal elements exist. Obviously, the rank of the PBH matrix of the state-space model remains as the full column matrix.

\subsection{Model predictive control}

Once the state estimator is established, the MPC method will be employed to optimize the input control sequence with the consideration of constraints, such as, state, output, and control (the induced electric current) for each element of a flexible EDT model explicitly [24-27].

Consider the high-fidelity model of EDT in the state-space form in (22). It can be discretized in the time domain by the zero-order hold method, such that,

$$
\begin{gathered}
\boldsymbol{X}_{k+1}=\boldsymbol{A}_{k}^{d} \boldsymbol{X}_{k}+\boldsymbol{B}_{k}^{d} \boldsymbol{u}_{k} \\
\boldsymbol{Y}_{k}=\boldsymbol{H}_{k}^{3} \boldsymbol{X}_{k}
\end{gathered}
$$

where $\boldsymbol{X}_{k}=\boldsymbol{X}_{o b s, k}^{+}$is the state of the tether estimated by the state estimator in the Section 3.1, $\boldsymbol{A}_{k}^{d}=e^{\boldsymbol{A}_{k}^{c} \Delta t}$ and $\boldsymbol{B}_{k}^{d}=\left(\boldsymbol{A}_{k}^{c}\right)^{-1}\left(e^{\boldsymbol{A}_{k}^{c} \Delta t}-\boldsymbol{E}_{3}\right) \boldsymbol{B}_{k}^{c}$ with $e^{(~)}$ and $\Delta t$ being the exponential function and sample time interval, respectively. In the current work, the exponential function $e^{(~)}$ is calculated by the Dgpadm algorithm [28], and the inverse of a matrix ()$^{-1}$ is calculated by the pseudoinverse algorithm.

By performing the recurrence computation of (39)-(40), the predicted state sequence $\underline{\boldsymbol{X}}_{k+1}$ can be 
written in a compact form as a function of the state $\boldsymbol{X}_{k}$ and the input state sequence $\underline{\boldsymbol{u}}_{k}$ at the current time $k$

$$
\begin{aligned}
& \underline{\boldsymbol{X}}_{k+1}=\underline{\boldsymbol{A}}_{k}^{d} \boldsymbol{X}_{k}+\underline{\boldsymbol{B}}_{k}^{d} \underline{\boldsymbol{u}}_{k} \\
& \underline{\boldsymbol{Y}}_{k+1}=\underline{\boldsymbol{H}}_{k+1} \underline{\boldsymbol{X}}_{k+1} \\
& \underline{\boldsymbol{X}}_{k+1}=\left[\begin{array}{c}
\boldsymbol{X}_{k+1 \mid k} \\
\boldsymbol{X}_{k+2 \mid k} \\
\mathbf{M} \\
\boldsymbol{X}_{k+n_{c} \mid k} \\
\mathbf{M} \\
\boldsymbol{X}_{k+n_{p} \mid k}
\end{array}\right], \underline{\boldsymbol{A}}_{k}^{d}=\left[\begin{array}{c}
\boldsymbol{A}_{k+0 \mid k}^{d} \\
\boldsymbol{A}_{k+1 \mid k}^{d} \boldsymbol{A}_{k+0 \mid k}^{d} \\
\mathrm{M} \\
\prod_{l=0}^{n_{c}-1} \boldsymbol{A}_{k+1 \mid k}^{d} \\
\mathrm{M} \\
\prod_{l=0}^{n_{p}-1} \boldsymbol{A}_{k+\mid k}^{d}
\end{array}\right], \underline{\boldsymbol{u}}_{k}=\left[\begin{array}{c}
\boldsymbol{u}_{k+0 \mid k} \\
\boldsymbol{u}_{k+1 \mid k} \\
\mathrm{M} \\
\boldsymbol{u}_{k+n_{c} \mid k} \\
\mathbf{M} \\
\boldsymbol{u}_{k+n_{c}-1 \mid k}
\end{array}\right] \\
& \underline{\boldsymbol{B}}_{k}^{d}=\left[\begin{array}{cccc}
\boldsymbol{B}_{k+0 \mid k}^{d} & \boldsymbol{O} & \mathrm{L} & \boldsymbol{0} \\
\boldsymbol{A}_{k+1 \mid k}^{d} \boldsymbol{B}_{k+0 \mid k}^{d} & \boldsymbol{B}_{k+1 \mid k}^{d} & \mathrm{~L} & \boldsymbol{0} \\
\mathrm{M} & \mathrm{M} & \mathrm{O} & \mathrm{M} \\
\prod_{j=1}^{n_{c}-1}\left(\boldsymbol{A}_{k+j \mid k}^{d}\right) \boldsymbol{B}_{k+0 \mid k}^{d} & \prod_{j=2}^{n_{c}-1}\left(\boldsymbol{A}_{k+j \mid k}^{d}\right) \boldsymbol{B}_{k+1 \mid k}^{d} & \mathrm{~L} & \boldsymbol{B}_{k+n_{c}-1 \mid k}^{d} \\
\mathrm{M} & \mathrm{M} & \mathrm{O} & \mathrm{M} \\
\prod_{j=1}^{n_{p}-1}\left(\boldsymbol{A}_{k+j \mid k}^{d}\right) \boldsymbol{B}_{k+0 \mid k}^{d} & \prod_{j=2}^{n_{p}-1}\left(\boldsymbol{A}_{k+j k k}^{d}\right) \boldsymbol{B}_{k+1 \mid k}^{d} & \mathrm{~L} & \sum_{l=n_{c}-1}^{n_{p}-2}\left[\prod_{j=l+1}^{n_{p}-1}\left(\boldsymbol{A}_{k+j k}^{d}\right) \boldsymbol{B}_{k+1 \mid k}^{d}\right]+\boldsymbol{B}_{k+n_{p}-1 \mid k}^{d}
\end{array}\right] \\
& \underline{\boldsymbol{Y}}_{k+1}=\left(\boldsymbol{Y}_{k+1 \mid k}, \boldsymbol{Y}_{k+2 \mid k}, \mathrm{~L}, \boldsymbol{Y}_{k+n_{c} \mid k}, \mathrm{~L}, \boldsymbol{Y}_{k+n_{p} \mid k}\right)^{T} \\
& \underline{\boldsymbol{H}}_{k+1}=\operatorname{diag}\left(\boldsymbol{H}_{k+1 \mid k}, \mathrm{~K}, \boldsymbol{H}_{k+n_{c} \mid k}, \mathrm{~K}, \boldsymbol{H}_{k+n_{p} \mid k}\right)
\end{aligned}
$$

where $\boldsymbol{H}_{k+j \mid k}=\boldsymbol{H}\left(j=1: n_{p}\right)$.

Substituting (41) into (42) leads to 


$$
\underline{\boldsymbol{Y}}_{k+1}=\underline{\boldsymbol{H}}_{k+1} \underline{\boldsymbol{A}}_{k}^{d} \boldsymbol{X}_{k}+\underline{\boldsymbol{H}}_{k+1} \underline{\boldsymbol{B}}_{k}^{d} \boldsymbol{u}_{k}
$$

The control objective is to minimize the difference between the actual state, or geometrical profile, of the tether $\boldsymbol{Y}(t) \in \mathbf{R}^{3 n}$ and the desired or reference state $\boldsymbol{Y}^{\text {ref }}(t) \in \mathbf{R}^{3 n}$ of a virtual straight tether that connects the main and sub spacecraft,

$$
\begin{gathered}
\min \boldsymbol{J}_{k}\left(\boldsymbol{X}_{k}, \underline{\boldsymbol{u}}_{k}, n_{p}, n_{c}\right)=\frac{1}{2}\left(\underline{\boldsymbol{Y}}_{k+1}-\underline{\boldsymbol{Y}}_{k+1}^{r e f}\right)^{T} \underline{\boldsymbol{Q}}_{m p c}\left(\underline{\boldsymbol{Y}}_{k+1}-\underline{\boldsymbol{Y}}_{k+1}^{r e f}\right)+\frac{1}{2} \underline{\boldsymbol{u}}_{k}^{T} \underline{\boldsymbol{P}}_{m p c} \underline{\boldsymbol{u}}_{k} \\
\underline{\boldsymbol{Q}}_{m p c}=\operatorname{diag}\left(\boldsymbol{Q}_{m p c}, \boldsymbol{Q}_{m p c}, \mathbf{K}, \boldsymbol{Q}_{m p c}\right) \in \mathbf{R}^{\left(n_{p} \cdot p\right) \times\left(n_{p} \cdot p\right)} \\
\underline{\boldsymbol{P}}_{m p c}=\operatorname{diag}\left(\boldsymbol{P}_{m p c}, \boldsymbol{P}_{m p c}, \mathbf{K}, \boldsymbol{P}_{m p c}\right) \in \mathbf{R}^{\left(n_{c} \cdot m\right) \times\left(n_{c} \cdot m\right)}
\end{gathered}
$$

subject to the system equation in (47) and the constraints

$$
\begin{gathered}
\underline{\boldsymbol{u}}_{k}^{\text {low }} \leq \underline{\boldsymbol{u}}_{k} \leq \underline{\boldsymbol{u}}_{k}^{u p p} \\
\underline{\boldsymbol{Y}}_{k+1}^{\text {low }} \leq \underline{\boldsymbol{Y}}_{k+1} \leq \underline{\boldsymbol{Y}}_{k+1}^{u p p}
\end{gathered}
$$

Here, the cost function $J_{k}$ at the current time $k$ is constructed based on the standard MPC procedure $[29,30], n_{P}$ and $n_{c}$ are the prediction and control horizons, respectively, $\boldsymbol{u}_{k}(t)$ is the controlled input, $\boldsymbol{Q}_{m p c} \in \mathbf{R}^{3 n \times 3 n}$ and $\boldsymbol{P}_{m p c} \in \mathbf{R}^{m \times m}$ are the output error weight and input control weight matrices, respectively, $\underline{\boldsymbol{u}}_{k}^{\text {low }}=\left(\boldsymbol{u}_{k}^{\text {low }}, \mathrm{K}, \boldsymbol{u}_{k}^{\text {low }}\right)^{T} \in \mathbf{R}^{n_{c} \cdot m}$ and $\underline{\boldsymbol{u}}_{k}^{\text {upp }}=\left(\boldsymbol{u}_{k}^{\text {upp }}, \mathrm{K}, \boldsymbol{u}_{k}^{\text {upp }}\right)^{T} \in \mathbf{R}^{n_{c} \cdot m}$ are the vectors of the lower and upper bounds of electric current in the tether at the current time, and $\underline{\boldsymbol{Y}}_{k+1}^{\text {low }}$ and $\underline{\boldsymbol{Y}}_{k+1}^{u p p}$ are the lower and upper bounds of the output state.

Substituting Eqs. (41)-(46) into Eq. (48) yields

$$
J_{k}\left(\boldsymbol{X}_{k}, \underline{\boldsymbol{u}}_{k}, n_{p}, n_{c}\right)=\frac{1}{2} \underline{\boldsymbol{u}}_{k}^{T} \underline{\boldsymbol{Z}}_{k}+\boldsymbol{F}^{T} \underline{\boldsymbol{u}}_{k}+\boldsymbol{S}_{m p c}^{T} \underline{\boldsymbol{Q}}_{m p c} \boldsymbol{S}_{m p c}
$$


where $\quad \boldsymbol{Z}=\left(\underline{\boldsymbol{B}}_{k}^{d}\right)^{T}\left(\underline{\boldsymbol{H}}_{k+1}\right)^{T} \underline{\boldsymbol{Q}}_{m p c} \underline{\boldsymbol{H}}_{k+1} \underline{\boldsymbol{B}}_{k}^{d}+\underline{\boldsymbol{P}}_{m p c} \quad, \quad \boldsymbol{F}=\left(\underline{\boldsymbol{B}}_{k}^{d}\right)^{T}\left(\underline{\boldsymbol{H}}_{k+1}\right)^{T} \underline{\boldsymbol{Q}}_{m p c}^{T} \boldsymbol{S}_{m p c} \quad$ with $\boldsymbol{S}_{m p c}=\left(\underline{\boldsymbol{H}}_{k+1}\right)^{T} \underline{\boldsymbol{A}}_{k}^{d} \boldsymbol{X}_{k}-\underline{\boldsymbol{Y}}_{k+1}^{r e f}$, and $\boldsymbol{S}_{m p c}^{T} \underline{\boldsymbol{Q}}_{m p c} \boldsymbol{S}_{m p c}$ is a constant term that does not affect the minimization of the cost function $[25,31]$. The superscript $T$ represents the transpose of a matrix.

Rewriting the input constraint in (51) in term of the vector $\underline{\boldsymbol{u}}_{k}$ leads to

$$
\begin{gathered}
\boldsymbol{D}_{1} \underline{\boldsymbol{u}}_{k} \leq \boldsymbol{P}_{1} \\
\boldsymbol{D}_{1}=\left[\begin{array}{c}
\boldsymbol{E}_{4} \\
-\boldsymbol{E}_{4}
\end{array}\right], \quad \boldsymbol{P}_{1}=\left[\begin{array}{l}
\underline{\boldsymbol{u}}_{k}^{\text {low }} \\
\underline{\boldsymbol{u}}_{k}^{\text {upp }}
\end{array}\right]
\end{gathered}
$$

where $\boldsymbol{D}_{1} \in \mathbf{R}^{2\left(n_{c} \cdot m\right)}$ and $\boldsymbol{P}_{1} \in \mathbf{R}^{2\left(n_{c} \cdot m\right)}$ are the mapping matrices, $\boldsymbol{E}_{4} \in \mathbf{R}^{n_{c} \cdot m}$ is an identity matrix, and $\underline{\boldsymbol{u}}_{k}^{\text {low }}=\left(\boldsymbol{u}_{k}^{\text {low }}, \mathbf{K}, \boldsymbol{u}_{k}^{\text {low }}\right)^{T} \in \mathbf{R}^{n_{c} \cdot m}$ and $\underline{\underline{u}}_{k}^{\text {upp }}=\left(\boldsymbol{u}_{k}^{\text {upp }}, \mathbf{K}, \boldsymbol{u}_{k}^{\text {upp }}\right)^{T} \in \mathbf{R}^{n_{c} \cdot m}$ are vectors of the lower and upper bounds of electric current in the tether at the current time $k$, respectively.

The state constraint in (52) is introduced to prevent a large departure of the predicted state $\underline{\boldsymbol{Y}}_{k+1}$ from the previous state to ensure a smooth control performance. This is achieved by defining the lower and upper bounds of the predicted vector as $\underline{\boldsymbol{Y}}_{k+1}^{\text {low }}=0.95 \underline{\boldsymbol{Y}}_{k+1}$ and $\underline{\boldsymbol{Y}}_{k+1}^{u p p}=1.05 \underline{\boldsymbol{Y}}_{k+1}$. Based on Eq. (47), Eq. (52) is reformulated in terms of the control vector $\underline{\boldsymbol{u}}_{k}$ as,

$$
\boldsymbol{D}_{2} \underline{\boldsymbol{u}}_{k} \leq \boldsymbol{P}_{2}
$$

where the matrix $\boldsymbol{D}_{2} \in \mathbf{R}^{2\left(n_{c} \times m\right)}$ and the vector $\boldsymbol{P}_{2} \in \mathbf{R}^{2\left(n_{c} \times m\right)}$ are formed as follows,

$$
\boldsymbol{D}_{2}=\left[\begin{array}{r}
\underline{\boldsymbol{H}}_{k+1} \underline{\boldsymbol{B}}_{k}^{d} \\
-\underline{\boldsymbol{H}}_{k+1} \underline{\boldsymbol{B}}_{k}^{d}
\end{array}\right], \quad \boldsymbol{P}_{2}=\left[\begin{array}{c}
\underline{\boldsymbol{Y}}_{k+1}^{u p p}-\underline{\boldsymbol{H}}_{k+1} \underline{\boldsymbol{A}}_{k}^{d} \boldsymbol{X}_{k} \\
-\underline{\boldsymbol{Y}}_{k+1}^{\text {low }}+\underline{\boldsymbol{H}}_{k+1} \underline{\boldsymbol{A}}_{k}^{d} \boldsymbol{X}_{k}
\end{array}\right]
$$

Combining Eqs. (54) and (56) by concatenating one on top of the other leads to a single constraint 
equation,

$$
\boldsymbol{D}_{3} \underline{\boldsymbol{u}}_{k} \leq \boldsymbol{P}_{3}
$$

where $\boldsymbol{D}_{3}=\left[\boldsymbol{D}_{1}^{T}, \boldsymbol{D}_{2}^{T}\right]^{T} \in \mathbf{R}^{4\left(n_{c} \times m\right)}$ and $\boldsymbol{P}_{3}=\left[\boldsymbol{P}_{1}^{T}, \boldsymbol{P}_{2}^{T}\right]^{T} \in \mathbf{R}^{4\left(n_{c} \times m\right)}$.

\subsection{Constraint softening}

Considering the fact that the EDT system is underactuated because there is only one control input (electric current), the constraint in Eq. (58) may occasionally be breached. Instead of imposing the hard constraint, which may lead to the infeasible hard-constrained problem, a constraint-softening technique is employed to penalize the constraint violation. Based on the slack variable method [32, 33], the problem in Eqs. (53) and (58) can be rewritten as,

$$
\begin{array}{ll}
\min & \boldsymbol{J}_{k}\left(\boldsymbol{X}_{k}, \underline{\boldsymbol{u}}_{k}, n_{p}, n_{c}\right)=\frac{1}{2} \underline{\boldsymbol{u}}_{k}^{T} \boldsymbol{Z} \underline{\boldsymbol{u}}_{k}+\boldsymbol{F}^{T} \underline{\boldsymbol{u}}_{k}+\boldsymbol{\varepsilon}_{1, k}^{T} \boldsymbol{P}_{4} \boldsymbol{\varepsilon}_{1, k} \\
\text { w.r.t } & \underline{\boldsymbol{u}}_{k} \\
\text { s.t } & \boldsymbol{D}_{3} \underline{\boldsymbol{u}}_{k} \leq \boldsymbol{P}_{3}+\boldsymbol{\varepsilon}_{1} \\
& \boldsymbol{0} \leq \boldsymbol{\varepsilon}_{1} \leq \boldsymbol{\varepsilon}_{1}^{u p p}
\end{array}
$$

where $\boldsymbol{\varepsilon}_{1} \in \mathbf{R}^{q_{2}}$ is the vector of slack variables denoting the constraint violation, $\boldsymbol{P}_{4} \in \mathbf{R}^{q_{2} \times q_{2}}$ is the positive-definite weight matrix for the slack variable vector $\varepsilon_{1}$, and $\varepsilon_{1}^{u p p}$ is a vector of the upper bound of the slack variables. When the base MPC problem is infeasible, the partial slack variables in Eq. (59) become positive to allow the constraints relaxed, which makes the problem feasible.

Introduce a new vector $\underline{\boldsymbol{u}}_{k}^{\prime}=\left(\underline{\boldsymbol{u}}_{k}, \boldsymbol{\varepsilon}_{1}\right)^{T}$. Substituting the new vector into Eq. (59) yields,

$$
\begin{array}{ll}
\min & J_{k}\left(\boldsymbol{X}_{k}, \underline{\boldsymbol{u}}_{k}^{\prime}, n_{c}, n_{p}\right)=\frac{1}{2} \underline{\boldsymbol{u}}_{k}^{\prime T} \boldsymbol{Z}^{\prime} \underline{\boldsymbol{u}}_{k}^{\prime}+\boldsymbol{F}^{\prime T} \underline{\boldsymbol{u}}_{k}^{\prime} \\
\text { w.r.t } & \underline{\boldsymbol{u}}_{k}^{\prime} \\
\text { s.t } & \boldsymbol{D}_{4} \underline{\boldsymbol{u}}_{k}^{\prime} \leq \boldsymbol{P}_{5}
\end{array}
$$


with

$$
\boldsymbol{Z}^{\prime}=\left[\begin{array}{cc}
\boldsymbol{Z} & \boldsymbol{0} \\
\boldsymbol{0} & \boldsymbol{P}_{4}
\end{array}\right], \quad \boldsymbol{F}^{\prime}=\left[\begin{array}{c}
\boldsymbol{F} \\
\boldsymbol{0}
\end{array}\right], \quad \boldsymbol{D}_{4}^{T}=\left[\begin{array}{cc}
\boldsymbol{D}_{3}^{T} & -\boldsymbol{E}_{5} \\
\boldsymbol{0} & \boldsymbol{E}_{5}
\end{array}\right], \quad \boldsymbol{P}_{5}=\left[\begin{array}{l}
\boldsymbol{P}_{4} \\
\boldsymbol{\varepsilon}_{1}^{u p p}
\end{array}\right]
$$

where $\boldsymbol{E}_{5} \in \mathbf{R}^{q_{2} \times q_{2}}$ is an identity matrix.

\subsection{Generation of reference state of tether}

The desired or reference state of tether is a straight line that passes through the main and subspacecraft. To ensure the length of the reference state is the same as the real tether, a special procedure is needed to map the nodes along the real tether to the reference tether see Fig. 3.

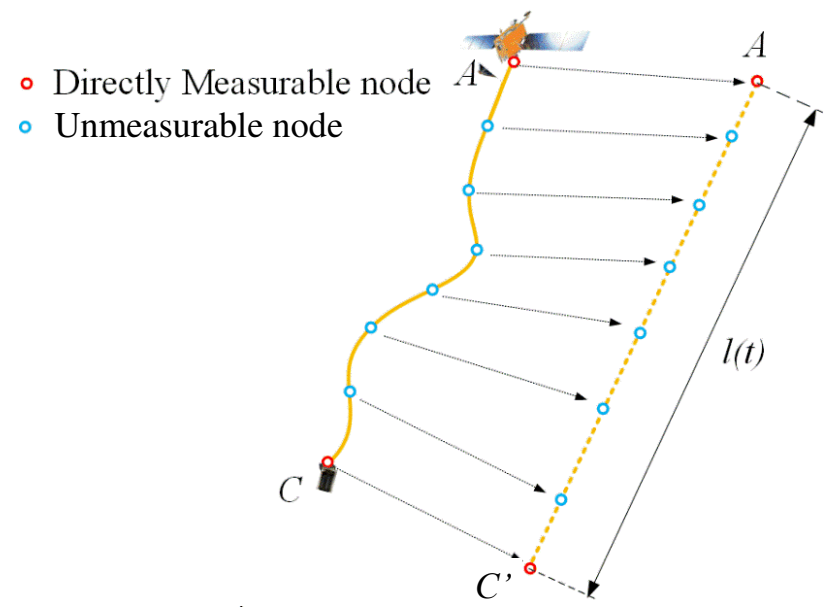

Fig. 3. Schematic diagram for generation of reference tether.

\section{(i) Generate the end nodes of reference tether}

First, generate a straight line from the main spacecraft (point A) to pass the sub-spacecraft (point C) and end at point $C^{\prime}$. The total length of the straight reference tether $\overline{A C^{\prime}}$ shall be the same as the instant length of the real tether $l(t)$, such that

$$
\boldsymbol{Y}_{A}^{r e f}=\boldsymbol{x}_{A}, \quad \boldsymbol{Y}_{C^{\prime}}^{r e f}=\boldsymbol{x}_{A}+l(t)\left(\boldsymbol{x}_{C}-\boldsymbol{x}_{A}\right) /\left\|\boldsymbol{x}_{C}-\boldsymbol{x}_{A}\right\|
$$


(ii) Generate the internal nodes along the reference tether

The coordinates of internal nodes of the real tether are mapped to the reference tether based on the instant length of each element one by one starting from the main spacecraft. Then, the position vector of all nodes of the reference tether is obtained,

$$
\boldsymbol{Y}^{r e f}=\left(\boldsymbol{Y}_{A}^{r e f}, \boldsymbol{Y}_{2}^{r e f}, \mathbf{K}, \boldsymbol{Y}_{n-1}^{r e f}, \boldsymbol{Y}_{C^{\prime}}^{r e f}\right)^{T} \in \mathbf{R}^{n_{p} \cdot 3 n}
$$

\subsection{Stability analysis of proposed MPC problem}

The asymptotic stability of MPC is examined through the monotonicity condition of cost function,

$$
J_{k+1}\left(\boldsymbol{X}_{k}, \underline{\boldsymbol{u}}_{k}^{\prime}, n_{c}, n_{p}\right) \leq J_{k}\left(\boldsymbol{X}_{k}, \underline{\boldsymbol{u}}_{k}^{\prime}, n_{c}, n_{p}\right)
$$

The monotonicity condition of the cost function of a constrained MPC is guaranteed if any of the following terminal conditions is applied (i) terminal equality constraints, (ii) terminal set constraints, and (iii) terminal penalty function [31, 32]. It is known that the terminal penalty function is superiority to the first two methods due to its wider feasible region [31, 34]. Accordingly, recasting the constrained MPC in Eq. (60) by adding a terminal inequality constraint, such that,

$$
\begin{array}{ll}
\min & J_{k}\left(\boldsymbol{X}_{k}, \underline{\boldsymbol{u}}_{k}^{\prime}, n_{c}, n_{p}\right)=\frac{1}{2} \underline{\boldsymbol{u}}_{k}^{\prime T} \boldsymbol{Z}^{\prime} \underline{\boldsymbol{u}}_{k}^{\prime}+\boldsymbol{F}^{\prime T} \underline{\boldsymbol{u}}_{k}^{\prime}+\left(\boldsymbol{Y}_{k+n_{p} \mid k}-\boldsymbol{Y}_{k+n_{p} \mid k}^{r e f}\right)^{T} \boldsymbol{Q}_{n_{p}}\left(\boldsymbol{Y}_{k+n_{p} \mid k}-\boldsymbol{Y}_{k+n_{p} \mid k}^{r e f}\right) \\
\text { w.r.t } & \underline{\boldsymbol{u}}_{k}^{\prime} \\
\text { s.t. } & \boldsymbol{D}_{4} \underline{\boldsymbol{u}}_{k}^{\prime} \leq \boldsymbol{P}_{5} \text { and } \boldsymbol{Y}_{k+n_{p} \mid k}-\boldsymbol{Y}_{k+n_{p} \mid k}^{r e f} \leq \boldsymbol{\varepsilon}_{2}
\end{array}
$$

where $\boldsymbol{\varepsilon}_{2} \in \mathbf{R}^{3 n}$ and $\boldsymbol{Q}_{N_{p}} \in \mathbf{R}^{3 n}$ is the penalty weight matrix that should satisfy the monotonicity condition of cost function [32], such that

$$
\boldsymbol{Q}_{n_{p}} \geq \boldsymbol{Q}_{m p c}+\boldsymbol{K}_{n p}^{T} \boldsymbol{P}_{m p c} \boldsymbol{K}_{n p}+\left(\boldsymbol{A}^{d}-\boldsymbol{B}^{d} \boldsymbol{K}_{n p}\right)^{T} \boldsymbol{Q}_{n_{p}}\left(\boldsymbol{A}^{d}-\boldsymbol{B}^{d} \boldsymbol{K}_{n p}\right)
$$

where $\boldsymbol{K}_{n p}$ is the optimal Gain of an LQR controller with the same $\boldsymbol{Q}_{m p c}$ and $\boldsymbol{P}_{m p c}$ weighting 
matrices, which is obtained by solving the corresponding Riccati equation [31, 32].

\subsection{Implementation of proposed MPC with FEKF estimator}

The proposed MPC with the FEKF estimator for controlling the geometric profile of a flexible EDT in the deorbit process is shown Fig. 4. The implementation process is show in Fig. 5. The constrained MPC problem is translated into a quadratic programming (QP) problem and solved by a QP solver.

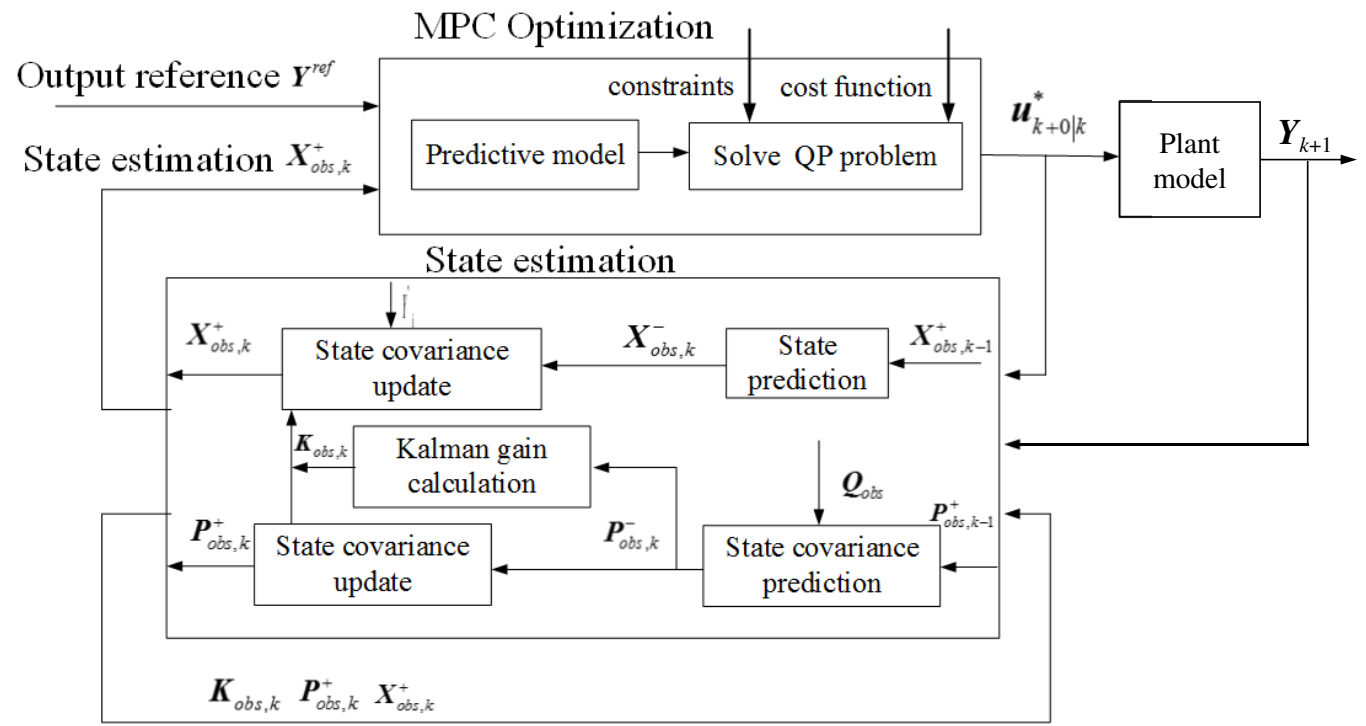

Fig. 4. Block diagram of the proposed MPC system with FEKF estimator. 


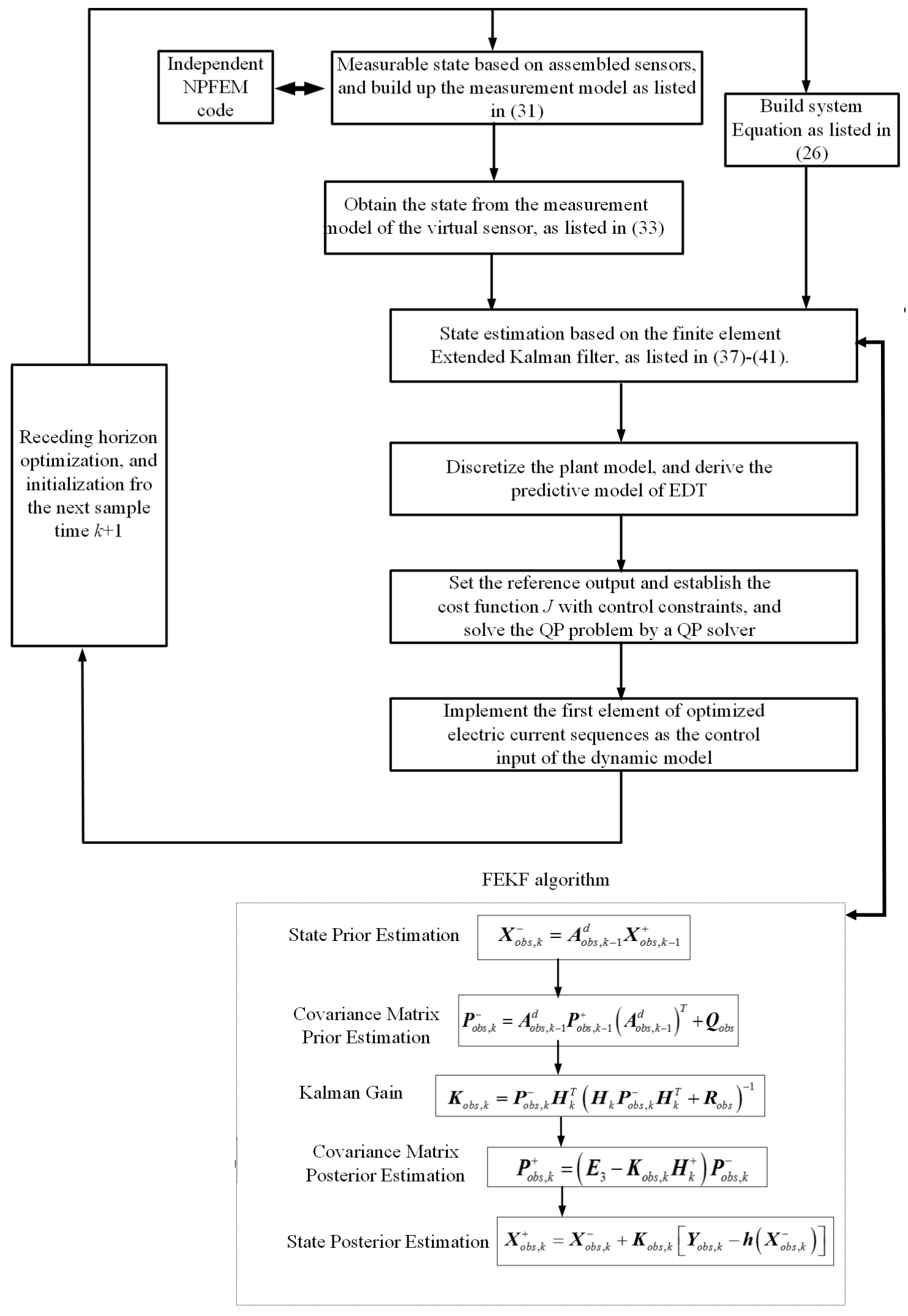

Fig. 5. Flowchart of implementation of MPC with FEKF estimator. 


\section{Numerical simulation}

\subsection{System parameters and initial conditions}

The effectiveness of the proposed MPC algorithm with FEKF estimator is demonstrated by numerical simulation. It is applied to control the geometric profile of an EDT in the deorbit process by regulating the induced electric current $I(s, t)$ in tether with two initial tether profiles as shown in Fig. 6, where the tether is initially straight (case A) and bent into a half circle (case B). As a comparison, each case is analyzed with and without control input by MPC method. The physical properties of the EDT are shown in Table I. The electrical boundary conditions, which represent the EDT system design, are given in Table II. The standard deviation $\boldsymbol{R}_{o b s}^{1}$ and $\boldsymbol{R}_{o b s}^{2}$ for the measurement model in (32) are assumed as $20 \mathrm{~m}$ as per [35]. The tether is uniformly divided into five elements, and the time step is set as $0.001 \mathrm{~s}$. The libration angles of the reference state are used to represent the libration motion of the discretized EDT model.

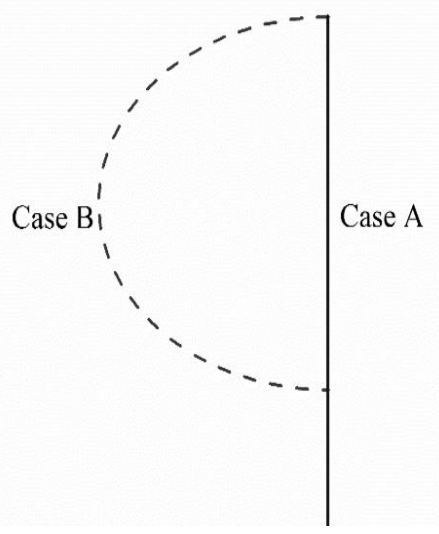

Fig. 6. Tether profile of an EDT of cases A and B.

The prediction and control horizons are selected as $n_{p}=20$ and $n_{c}=5$, and the weight matrices are set as $\boldsymbol{P}_{m p c}=1000 \boldsymbol{E}_{2}$ and $\boldsymbol{Q}_{m p c}=0.1 \boldsymbol{E}_{2}$ to make the tracking error as a primary optimization goal, 
based on a priori knowledge $[25,36]$ and the trade-off between tracking error and control effect.

Table 1 Physical properties of EDT [4]

\begin{tabular}{lc}
\hline Name & Value \\
\hline Mass of Main/Sub Spacecraft $(\mathrm{kg})$ & $85.0 / 0.02$ \\
Dimension of Main Spacecraft $(\mathrm{L} \times \mathrm{W} \times \mathrm{H})(\mathrm{m})$ & $0.5 \times 0.5 \times 0.2$ \\
Dimension of Sub Spacecraft $(\mathrm{L} \times \mathrm{W} \times \mathrm{H})(\mathrm{m})$ & $0.01 \times 0.01 \times 0.01$ \\
Length of Tether $(\mathrm{m})$ & 675.0 \\
Density of Tether $\left(\mathrm{kg} / \mathrm{m}^{3}\right)$ & $2,700.0$ \\
Diameter of Tether $(\mathrm{m})$ & 0.0005 \\
Elastic Module of Tether $(\mathrm{MPa})$ & 72.0 \\
Initial Altitude $(\mathrm{km})$ & 450.0 \\
Orbital Inclination Angle $(\mathrm{deg})$. & 0.0 \\
\hline
\end{tabular}

Table 2 Boundary condition of OML theory [4]

\begin{tabular}{l|c}
\hline Name & Value \\
\hline Potential Bias of Emitter Gate $\Phi_{P W}(V)$ & 60.0 \\
Potential Bias of Battery $\Phi_{C H E}(V)$ & 60.0 \\
Variable Resistance $Z_{T}$ (initial) $(\Omega)$ & 0.0 \\
\hline
\end{tabular}

The lower and upper bounds of the input current in Eq. (58) is defined as zero and the maximum possible induced electric current respectively. This choice of the upper bound of input current is to maximize the electron collection efficiency of the bare tether $[4,18]$. The maximum possible induced electric current is determined by solving the OML theory in Eq. (17) with the boundary conditions in Table II [4] with the current orbital position of the EDT.

\subsection{Case A}

First, the dynamic simulation is conducted without any control by (1), where the electric current is obtained by numerically solving (17) [18]. The numerical simulation stops once the libration 
angles of the EDT exceed 90 degrees, which implies the tether flips over. The simulation results are shown in Figs. 8-10. Second, the dynamic simulation is conducted with the MPC in (60). With the control input of electric current at the end points of tether (first and last nodes), i.e., $\boldsymbol{u}=\left(I_{1}, I_{n}\right)^{T} \in \mathbf{R}^{m=2}$, the maximum possible electric current $\boldsymbol{u}^{*}=\left(I_{1}^{*}, I_{n}^{*}\right)^{T}$ is obtained by solving the OML theory. Then, the value of electric current at the internal points of tether $\left(I_{2}, \mathrm{~L}, I_{n-1}\right)^{T}$ is re-calculated by feeding $\boldsymbol{u}^{*}=\left(I_{1}^{*}, I_{n}^{*}\right)^{T}$ into the OML theory. The calculated values are then used as the upper bound of the input control in Eq. (60). The simulation results are plotted in Figs. 7-9 for comparison.

The simulation results in Fig. 7 shows the in-plane libration angle of the EDT with/without MPC quickly exceeds $90^{\circ}(\approx 1.57 \mathrm{rad})$ because the MPC does not include the constraints on the libration angles. The EDT flips over and becomes unstable see Fig. 8. This is expected because there is no control for the libration angle. The Lorentz force keeps pumping energy into the libration motion of the EDT system. However, it is interesting to see in Fig. 8 that the profile of the EDT is much closer to the straight virtual tether when the proposed MPC is applied. The application of MPC results in a longer stable period of time before flipping over than the case without electric current control, see Fig. 8. The electric current at the cathodic end of EDT is shown in Fig. 9. It shows the induced electric current in the EDT is at the maximum level in the case without control as designed, see Fig. 9(a). The profile of the electric current with MPC is shown in Fig. 9(b), where the profile of electric current is clearly regulated between the minimum value (zero) and the upper bound. This explains why the profile of tether with control is closer to the straight tether and the stable period is about $15 \%$ longer than the case without electric current control. 

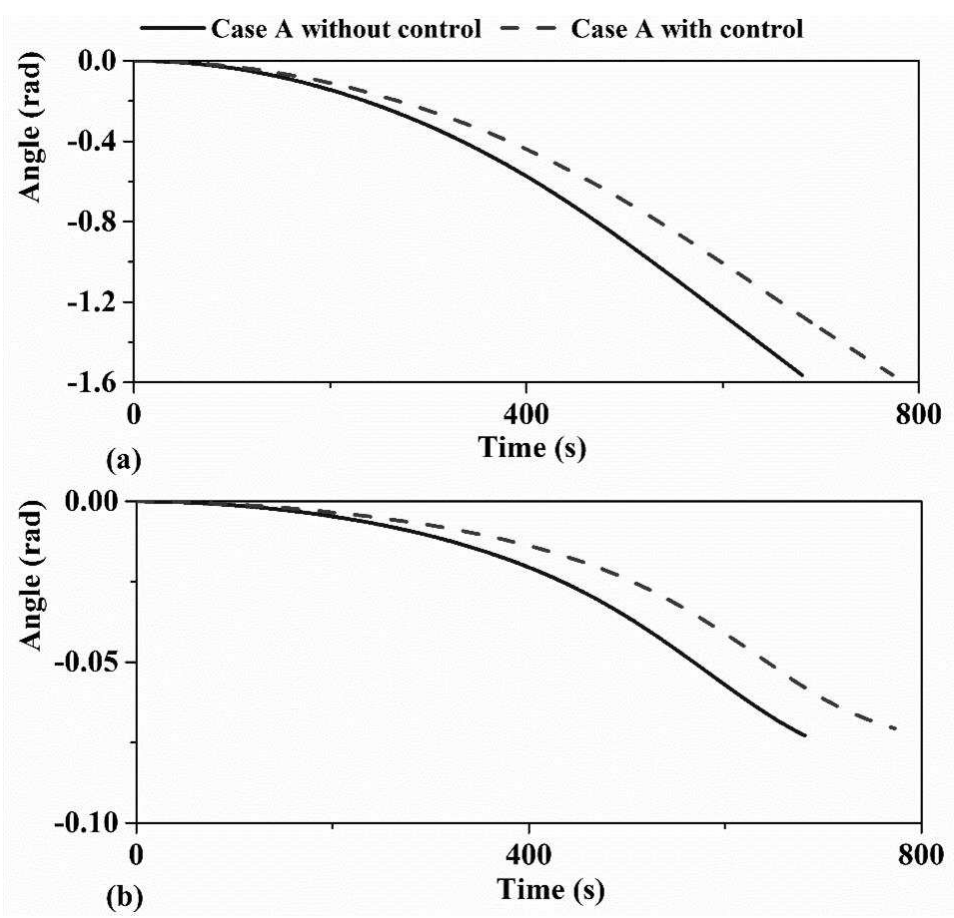

Fig. 7. Libration angles of EDT at case A (a) in-plane, (b) out-of-plane. 

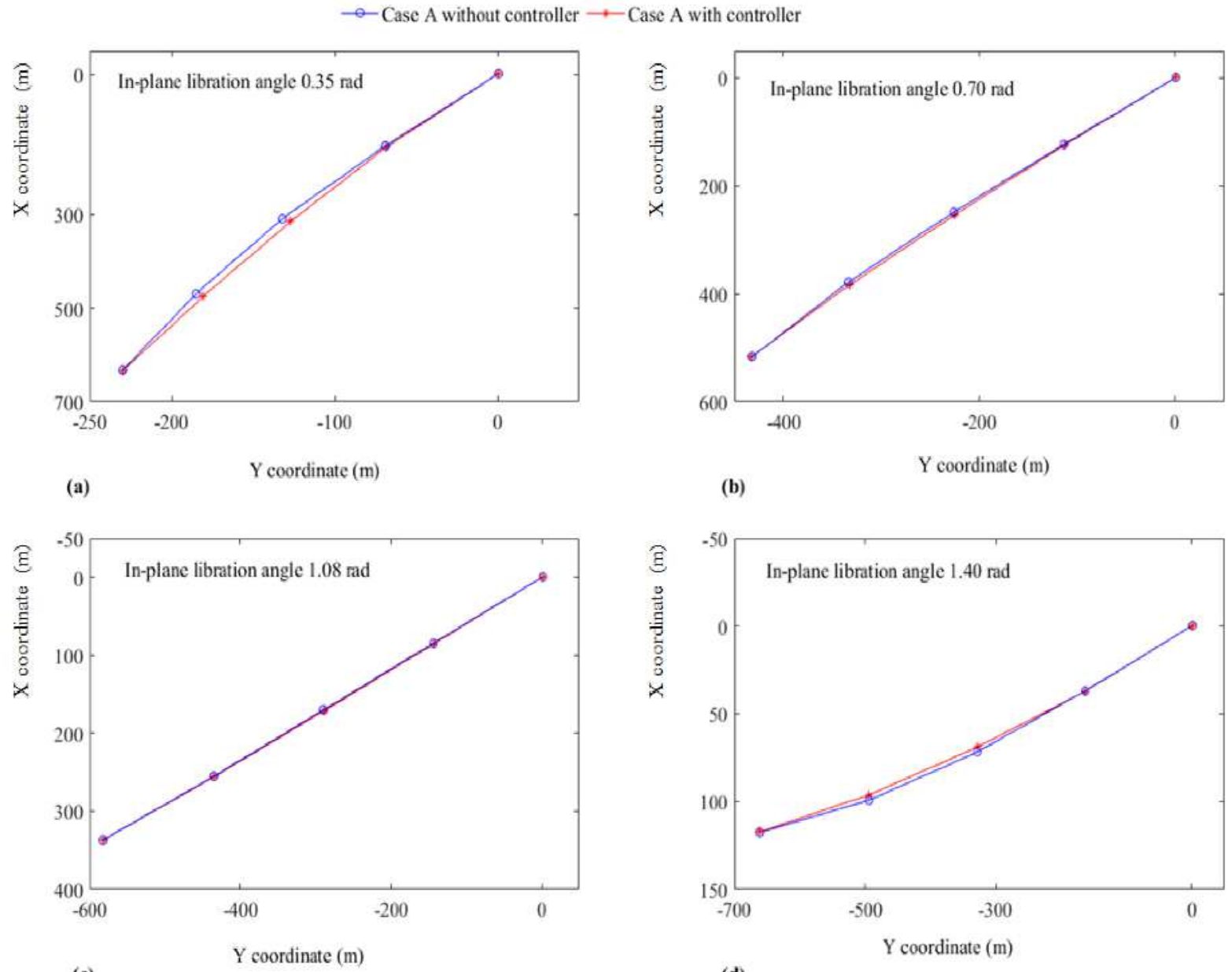

(d)

Fig. 8. Geometric configuration of the flexible EDT at case A.
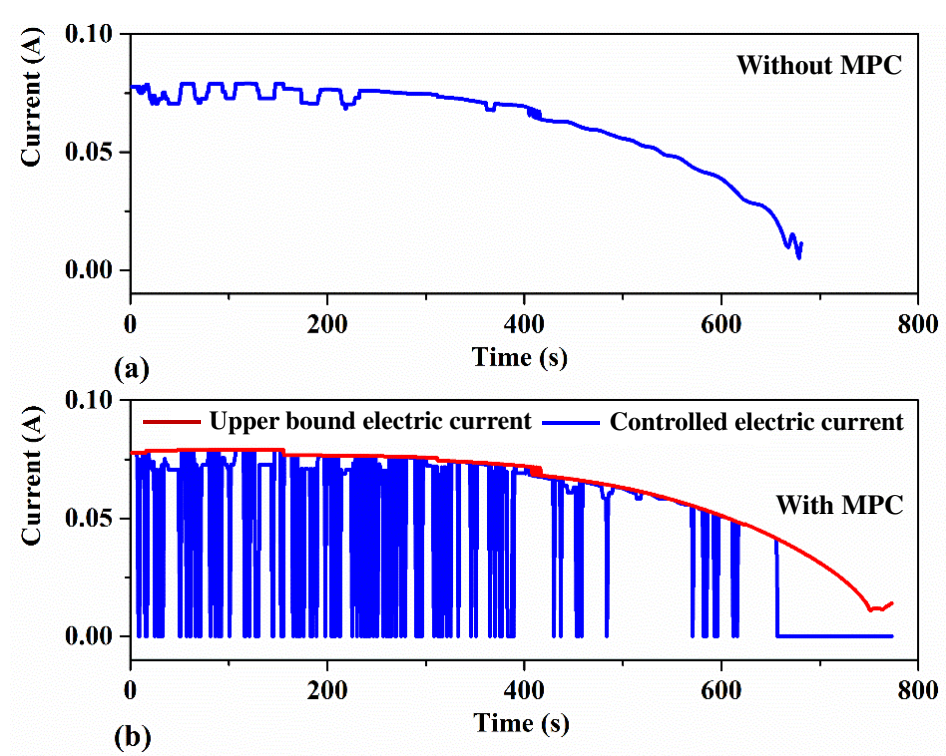

Fig. 9. Case A: Electric current at cathodic end. 


\subsection{Case B}

In this case, the tether is initially bent into a half-circle shape, and both ends are in the local vertical.

Similarly, two simulations are conducted with and without MPC until the tether flips over.

Simulation results are shown in Figs. 10-12.
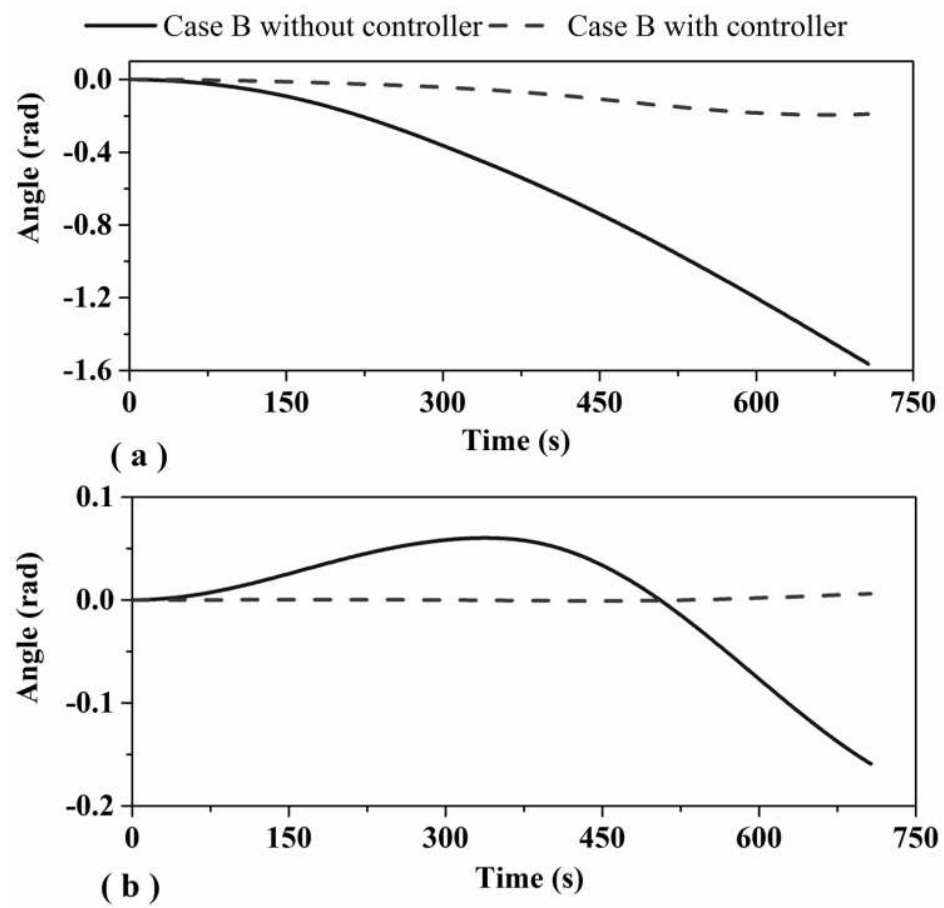

Fig. 10. Libration angle of EDT at case B (a) in-plane, (b) out-of-plane. 


\section{- Case B without controller $\rightarrow$ Case B with controller}
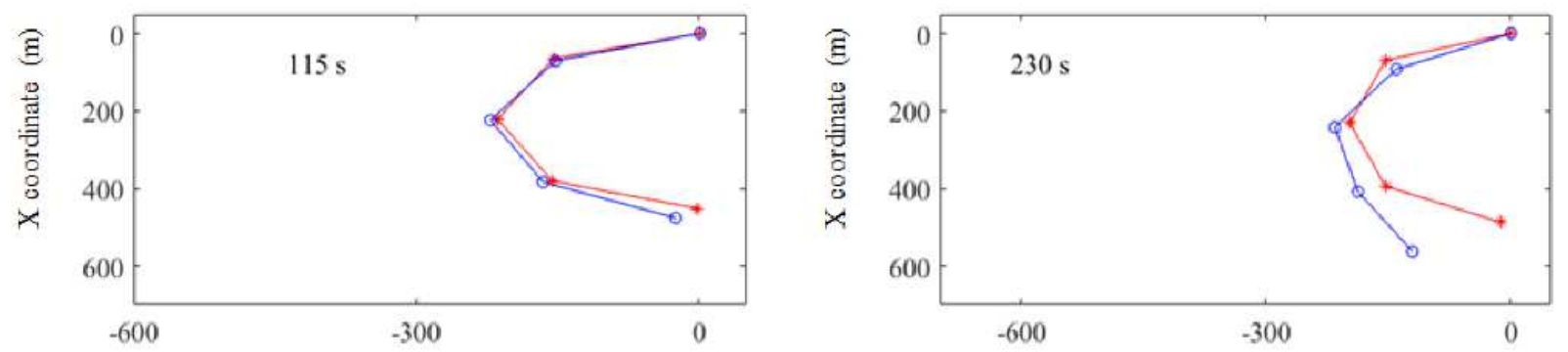
(a)
Y coordinate $(\mathrm{m})$

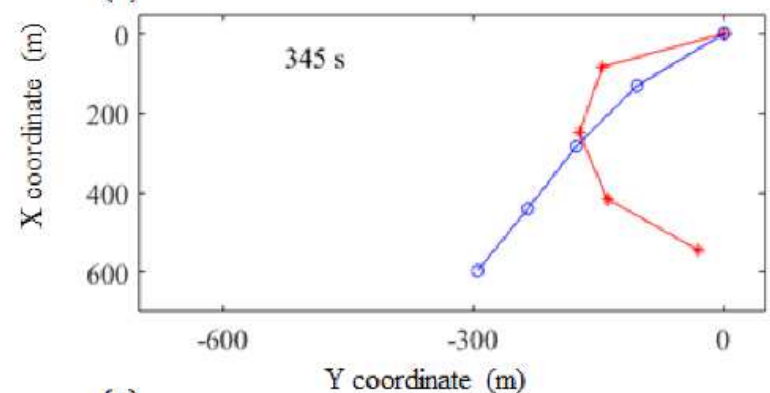

(b)

Y coordinate $(\mathrm{m})$

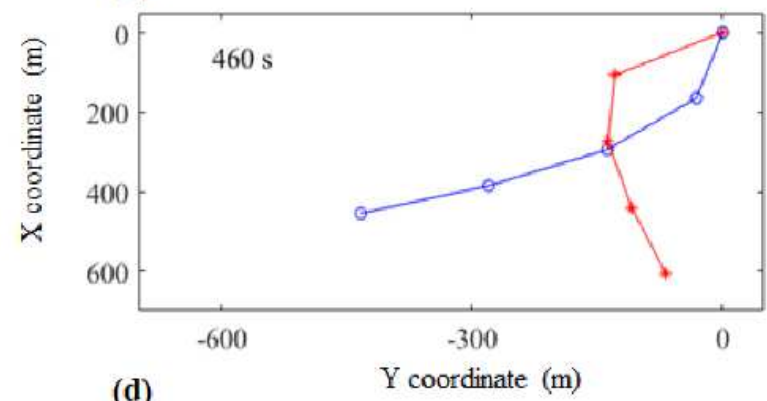

(c)

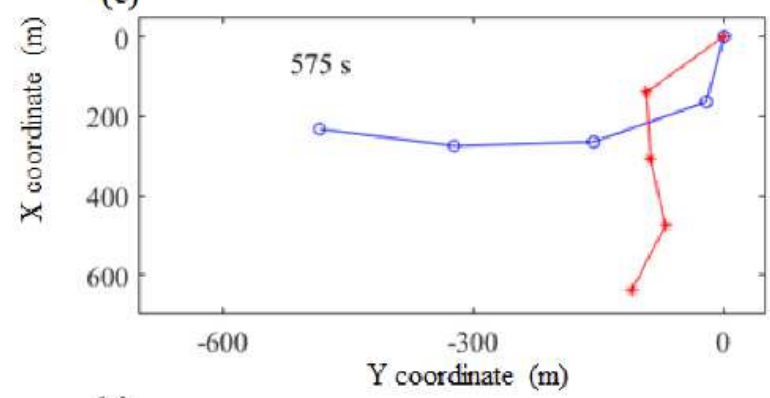

(e)

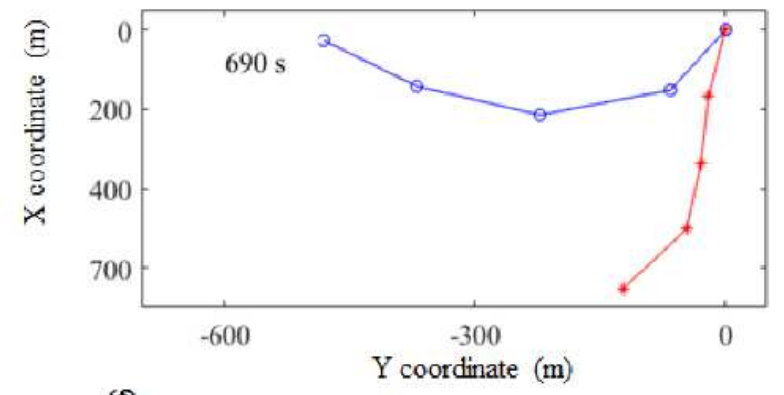

(f)

Fig. 11. Geometric configuration of the flexible EDT at case B. 

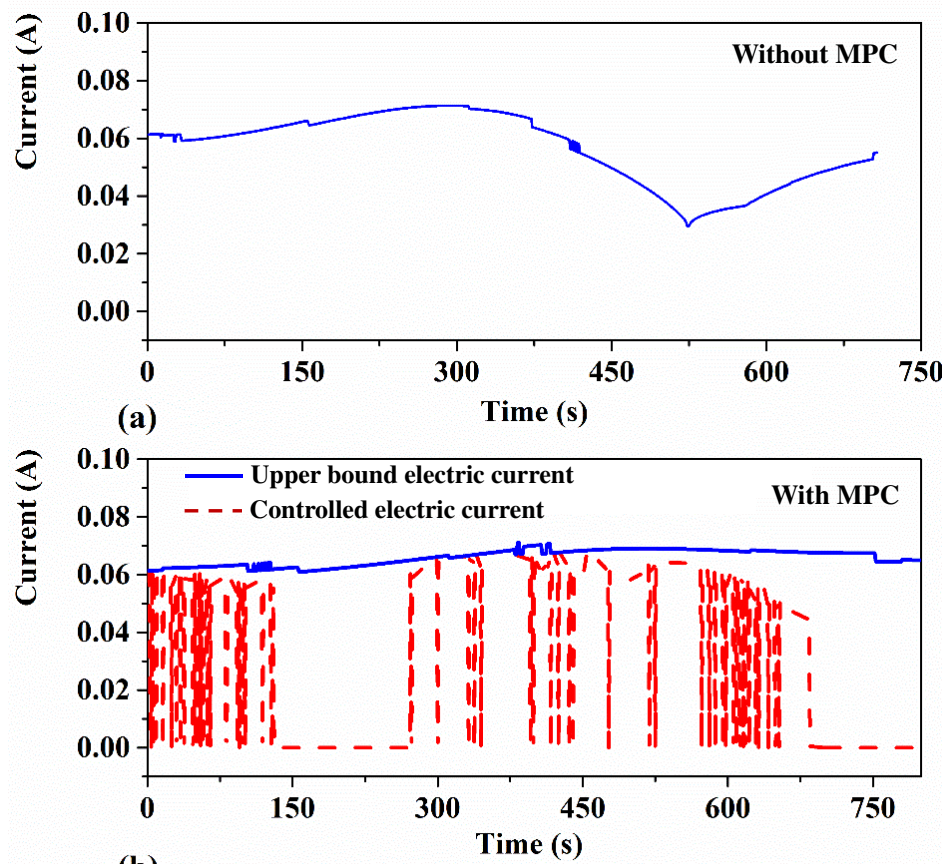

(b)

Fig. 12. Case B: Electric current at cathodic end.

Figure 10 shows the libration motion of EDT for the Case B. It clearly shows that the in-plane angle of EDT without tether profile control quickly flips over $90^{\circ}$ as expected due to no libration angle control. On the contrary, the in-plane angle of EDT with tether profile control is significantly less when the proposed controller is applied. For instance, the in-plane angle of EDT with the electric current regulation by the MPC is less than $0.2 \mathrm{rad}$, while the in-plane angle of EDT without the electric current control reaches $90^{\circ}$ in the same time period. This demonstrates the proposed MPC effectively straightens the tether towards the straight reference state in the local vertical initially and results in a much smaller in-plane libration angle, see Fig. 10(b) and 11. Finally, Fig. 12 shows the electric current profile at the cathodic end is significantly different in cases of with and without MPC because the corresponding tether profiles are very different. This is very different from the Case A where the profiles of tether in both controlled and uncontrolled conditions are similar. In summary, the proposed MPC with FEKF estimator is capable of 
straightening the bent flexible tether in the libration motion.

\section{Conclusion}

In this paper, a model predictive control (MPC) with finite element Kalman filter (FEKF) estimator is proposed to straighten a bent flexible electrodynamic tether or prevent a straight electrodynamic tether from bending in the deorbit process. This is achieved by controlling the induced electric current in the tether. The non-measurable tether profile is effectively obtained by the proposed FEKF estimator with the input of the measured state at both ends of the tether. By tracking a virtual straight tether that passes through both ends of tether as the reference state, the framework of the finite element based the model predictive control algorithm, which includes the Orbital-MotionLimited theory and constraints on the input electric current and the control errors, is developed. Moreover, the stability of the proposed MPC and the observability of the state estimator are examined analytically. The problem of controlling the geometric profile of the electrodynamic tether is transferred into a trajectory tracking problem and then solved by nonlinear programming. Numerical case studies successfully demonstrate that the proposed model predictive control method with FEKF estimator is effective in controlling the geometric profile of the electrodynamic tether towards the straight reference state with a single control input of electric current.

\section{Declarations}

\subsection{Funding}

This work is supported by the Discovery Grant (RGPIN-2018-05991) and Discovery Accelerator Supplements Grant (RGPAS-2018-522709) of Natural Sciences and Engineering Research Council of Canada. 
6.2 Conflicts of interest/Competing interests

The authors declare no conflict of interest in this article.

6.3 Availability of data and material

Data sharing not applicable to this article as no datasets were generated or analyzed during the current study.

6.4 Code availability (software application or custom code)

The custom codes in the current study are available from the corresponding author on reasonable request.

6.4 Authors' contributions

Dr. Gangqiang Li

Conceptualization: Supporting; Formal analysis: Equal; Investigation: Equal; Methodology: Equal; Software: Lead; Validation: Equal; Writing - original draft: Equal; Writing - review \& editing: Supporting.

Dr. Zheng H. Zhu

Conceptualization: Lead; Formal analysis: Lead; Funding acquisition: Lead; Investigation: Lead; Methodology: Lead; Project administration: Lead; Resources: Lead; Software: Supporting; Supervision: Lead; Validation: Equal; Writing - original draft: Equal; Writing - review \& editing: Lead. 


\subsection{Ethics approval}

Not applicable.

6.6 Consent to participate

Not applicable.

6.7 Consent for publication

Not applicable.

\section{References}

[1] Shan, M., Guo J., Gill E., Review and comparison of active space debris capturing and removal methods, Progress in Aerospace Sciences, 80 (January), 8018-32 (2016). https://doi.org/10.1016/j.paerosci.2015.11.001

[2] Sanmartin, J.R., Lorenzini E.C., Martinez-Sanchez M., Electrodynamic Tether Applications and Constraints, Journal of Spacecraft and Rockets, 47(3), 442-456 (2010). https://doi.org/10.2514/1.45352

[3] Li, G., Zhu Z.H., Multiphysics Finite Element Modeling of Current Generation of Bare Flexible Electrodynamic Tether, Journal of Propulsion and Power, 33(2), 408-419 (2017). https://doi.org/10.2514/1.b36211

[4] Li, G., Zhu Z.H., Parameter influence on electron collection efficiency of a bare electrodynamic tether, Science China Information Sciences, 61(2), 022201:1-022201:12 (2017). https://doi.org/10.1007/s11432-017-9219-1 
[5] Li, G., Zhu Z.H., Ruel S., Meguid S.A., Multiphysics elastodynamic finite element analysis of space debris deorbit stability and efficiency by electrodynamic tethers, Acta Astronautica, 137 (August), 320-333 (2017). https://doi.org/10.1016/j.actaastro.2017.04.025

[6] Corsi, J., Iess L., Stability and control of electrodynamic tethers for de-orbiting applications, Acta Astronautica, 48(5), 491-501 (2001). https://doi.org/10.1016/S0094-5765(01)00049-2

[7] Williams, P., Electrodynamic Tethers Under Forced-Current Variations Part II: Flexible-Tether Estimation and Control, Journal of Spacecraft and Rockets, 47(2), 320-333 (2010). https://doi.org/10.2514/1.45733

[8] Wen, H., Yu B., Jin D., Energy-Based Current Control for Stabilizing a Flexible Electrodynamic Tether System, Journal of Beijing Institute of Technology, 60(1), 1-4 1(2017). https://doi.org/10.15918/j.jbit1004-0579.201726.0101

[9] Tortora, P., Somenzi L., Iess L., Licata R., Small mission design for testing in-orbit an electrodynamic tether deorbiting system, Journal of Spacecraft and Rockets, 43(4), 883-892 (2006). https://doi.org/10.2514/1.15359

[10] Tragesser, S.G., San H., Orbital maneuvering with electrodynamic tethers, Journal of Guidance, Control, and Dynamics, 26(5), 805-810 (2003). https://doi.org/10.2514/2.5115

[11] Li, G., Zhu Z.H., Cain J., Newland F., Czekanski A., Libration Control of Bare Electrodynamic Tethers Considering Elastic-Thermal-Electrical Coupling, Journal of Guidance, Control, and Dynamics, 39(3), 642-654 (2016). https://doi.org/10.2514/1.g001338

[12] Li, G., Zhu Z.H., Estimation of Flexible Space Tether State based on End Measurement by Finite Element Kalman Filter State Estimator, Advances in Space Research, 67(10) 3282-3293 (2021). https://doi.org/10.1016/j.asr.2021.01.057

[13] Sanmartin, J.R., Martinez-Sanchez M., Ahedo E., Bare wire anodes for electrodynamic 
tethers, Journal of Propulsion and Power, 9(3), 353-360 (1993). 10.2514/3.23629

[14] Li, G., Zhu Z.H., Meguid S.A., Libration and transverse dynamic stability control of flexible bare electrodynamic tether systems in satellite deorbit, Aerospace Science and Technology, 49(Februrary), 112-129 (2016). https://doi.org/10.1016/j.ast.2015.11.036

[15] Wang, C., Li G., Zhu Z.H., Li A., Mass Ratio of Electrodynamic Tether to Spacecraft on Deorbit Stability and Efficiency, Journal of Guidance, Control, and Dynamics, 39(9), 21922198 (2016). https://doi.org/10.2514/1.g000429

[16] Sun, F.J., Zhu Z.H., LaRosa M., Dynamic modeling of cable towed body using nodal position finite element method, Ocean Engineering, 38(4), 529-540 (2011). https://doi.org/10.1016/j.oceaneng.2010.11.016

[17] Li, G.Q., Zhu Z.H., Long-term dynamic modeling of tethered spacecraft using nodal position finite element method and symplectic integration, Celestial Mechanics and Dynamical Astronomy, 123(4), 363-386 (2015). https://doi.org/10.1007/s10569-015-9640-5

[18] Li, G., Zhu Z.H., Precise Analysis of Deorbiting by Electrodynamic Tethers Using Coupled Multiphysics Finite Elements, Journal of Guidance, Control, and Dynamics, 40(12), 3348-3357 (2017). https://doi.org/10.2514/1.g002738

[19] Reif, K., Unbehauen R., The extended Kalman filter as an exponential observer for nonlinear systems, IEEE Transactions on Signal Processing, 47(8), 2324-2328 (1999). https://doi.org/10.1109/78.774779

[20] Babacan, E.K., Ozbek L., Efe M., Stability of the Extended Kalman Filter When the States are Constrained, IEEE Transactions on Automatic Control, 53(11), 2707-2711 (2008). https://doi.org/10.1109/TAC.2008.2008333

[21] Sun, X., Zhang H., Cai Y., Wang S., Chen L., Hybrid modeling and predictive control of 
intelligent vehicle longitudinal velocity considering nonlinear tire dynamics, Nonlinear Dynamics, 97(2), 1051-1066 (2019). https://doi.org/10.1007/s11071-019-05030-5

[22] Williams, R.L., Lawrence D.A., Linear state-space control systems, Wiley Online Library, 2007 2007, pp 174-176.

[23] van de Walle, A., Naets F., Desmet W., Virtual microphone sensing through vibro-acoustic modelling and Kalman filtering, Mechanical Systems and Signal Processing, 104, 120-133 (2018). https://doi.org/10.1016/j.ymssp.2017.08.032

[24] Franchi, L., Feruglio L., Mozzillo R., Corpino S., Model predictive and reallocation problem for CubeSat fault recovery and attitude control, Mechanical Systems and Signal Processing, 98, 1034-1055 (2018). https://doi.org/10.1016/j.ymssp.2017.05.039

[25] Li, P., Zhu Z.H., Line-of-sight nonlinear model predictive control for autonomous rendezvous in elliptical orbit, Aerospace Science and Technology, 69(October), 236-243 (2017). https://doi.org/10.1016/j.ast.2017.06.030

[26] Zhu, M., Chen H., Xiong G., A model predictive speed tracking control approach for autonomous ground vehicles, Mechanical Systems and Signal Processing, 87, 138-152 (2017). https://doi.org/10.1016/j.ymssp.2016.03.003

[27] Zhang, P., Wu Z., Meng Y., Tan M., Yu J., Nonlinear model predictive position control for a tail-actuated robotic fish, Nonlinear Dynamics, 101(4), 2235-2247 (2020). https://doi.org/10.1007/s11071-020-05963-2

[28] Sidje, R.B., Expokit: A software package for computing matrix exponentials, ACM Transactions on Mathematical Software (TOMS), 24(1), 130-156 (1998). https://doi.org/10.1145/285861.285868

[29] Kassaeiyan, P., Tarvirdizadeh B., Alipour K., Control of tractor-trailer wheeled robots 
considering self-collision effect and actuator saturation limitations, Mechanical Systems and Signal Processing, 127, 388-411 (2019). https://doi.org/10.1016/j.ymssp.2019.03.016

[30] Li, D., Cao H., Zhang X., Chen X., Yan R., Model predictive control based active chatter control in milling process, Mechanical Systems and Signal Processing, 128, 266-281 (2019). https://doi.org/10.1016/j.ymssp.2019.03.047

[31] Haghighat, S., Liu H.H.T., Martins J.R.R.A., Model-Predictive Gust Load Alleviation Controller for a Highly Flexible Aircraft, Journal of Guidance, Control, and Dynamics, 35(6), 1751-1766 (2012). https://doi.org/10.2514/1.57013

[32] Rawlings, J.B., Mayne D.Q., Diehl M., Model predictive control: theory, computation, and design, Nob Hill Publishing Madison, WI, 2017.

[33] Zong, L., Luo J., Wang M., Yuan J., Obstacle avoidance handling and mixed integer predictive control for space robots, Advances in Space Research, 61(8), 1997-2009 (2018). https://doi.org/10.1016/j.asr.2018.01.025

[34] Wen, H., Jin D., De-spinning of tethered space target via partially invariable deployment with tension control, Nonlinear Dynamics, 96(1), 637-645 (2019). https://doi.org/10.1007/s11071019-04811-2

[35] Wen, H., Zhu Z.H., Jin D., Hu H., Model Predictive Control with Output Feedback for a Deorbiting Electrodynamic Tether System, Journal of Guidance, Control, and Dynamics, 39(6), 2451-2456 (2016). https://doi.org/10.2514/1.G000535

[36] Li, P., Zhu Z.H., Model predictive control for spacecraft rendezvous in elliptical orbit, Acta Astronautica, 146(May),339-348 (2018). https://doi.org/10.1016/j.actaastro.2018.03.025 
Figures

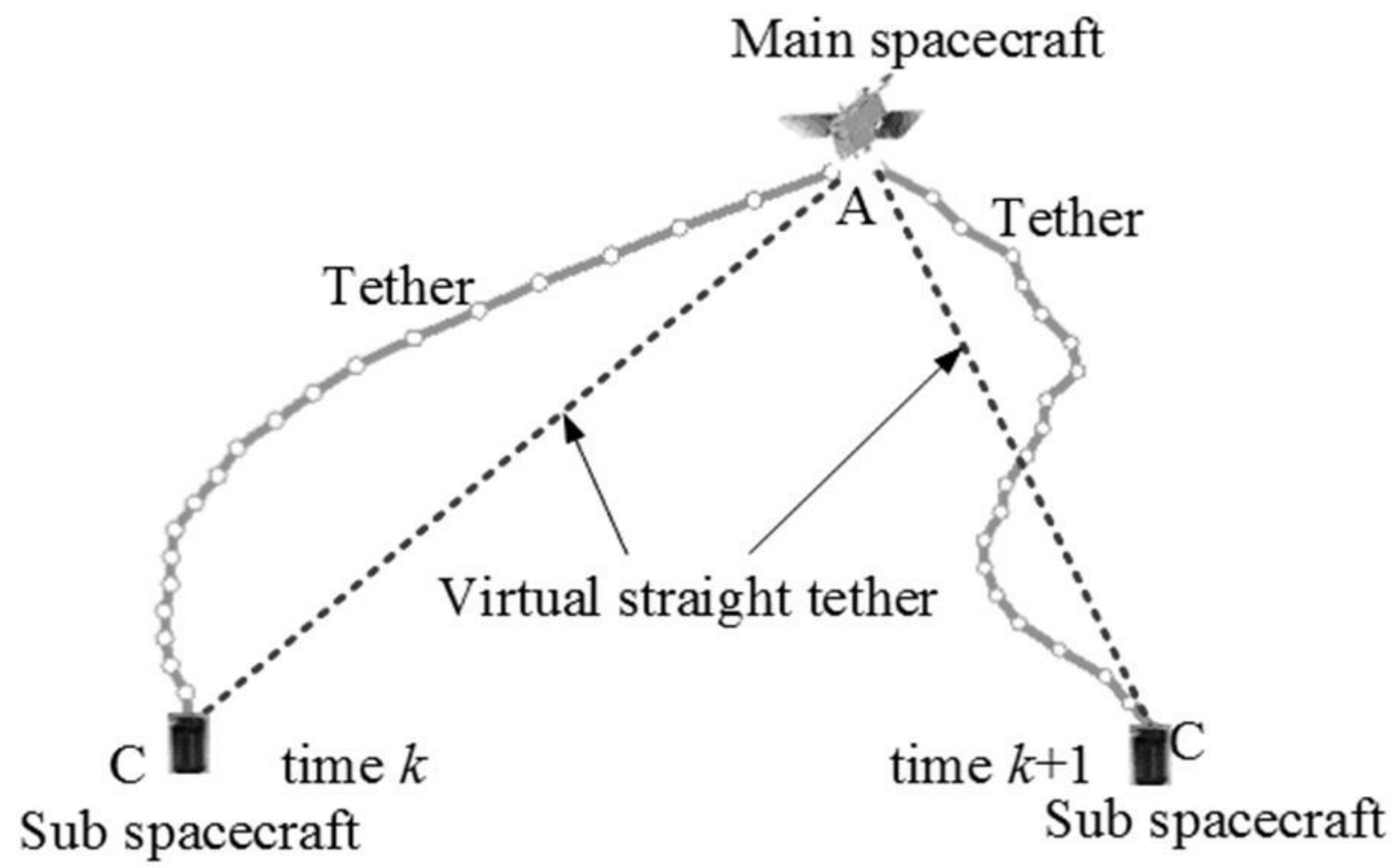

Figure 1

Schematic diagram of a discretized EDT with virtual straight tethers. 


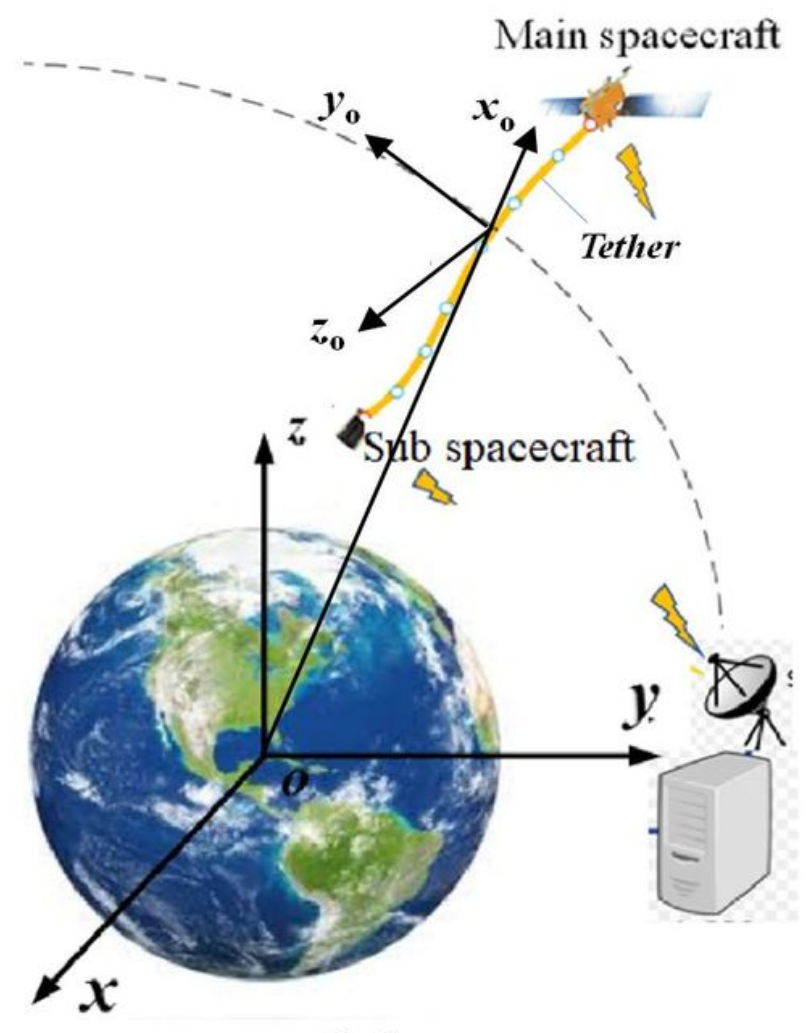

(a)

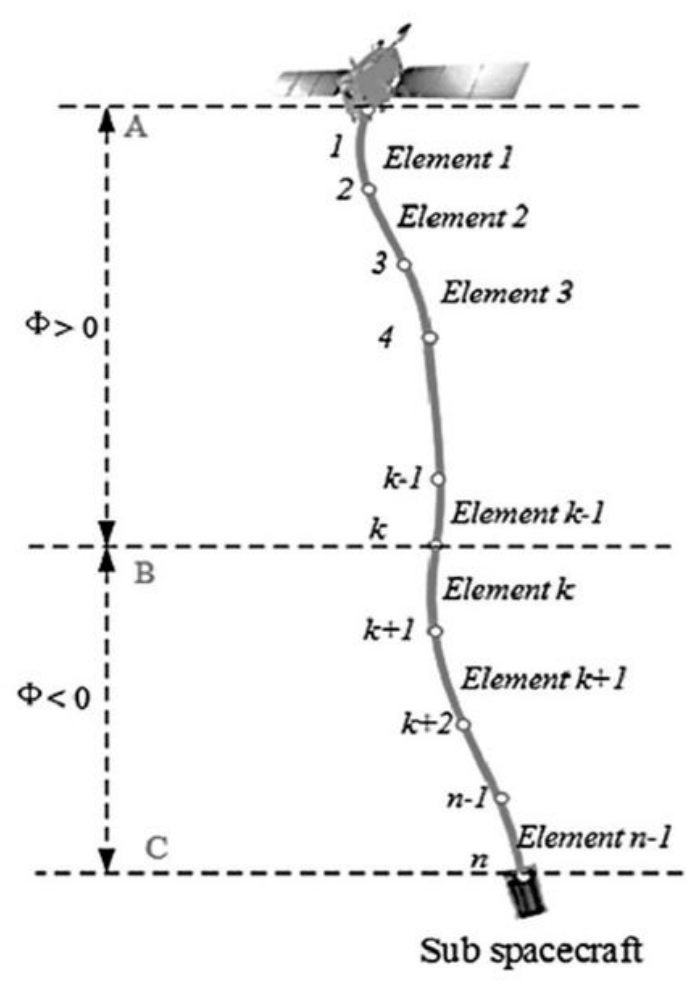

(b)

Figure 2

The schematic diagram of a flexible EDT model. 


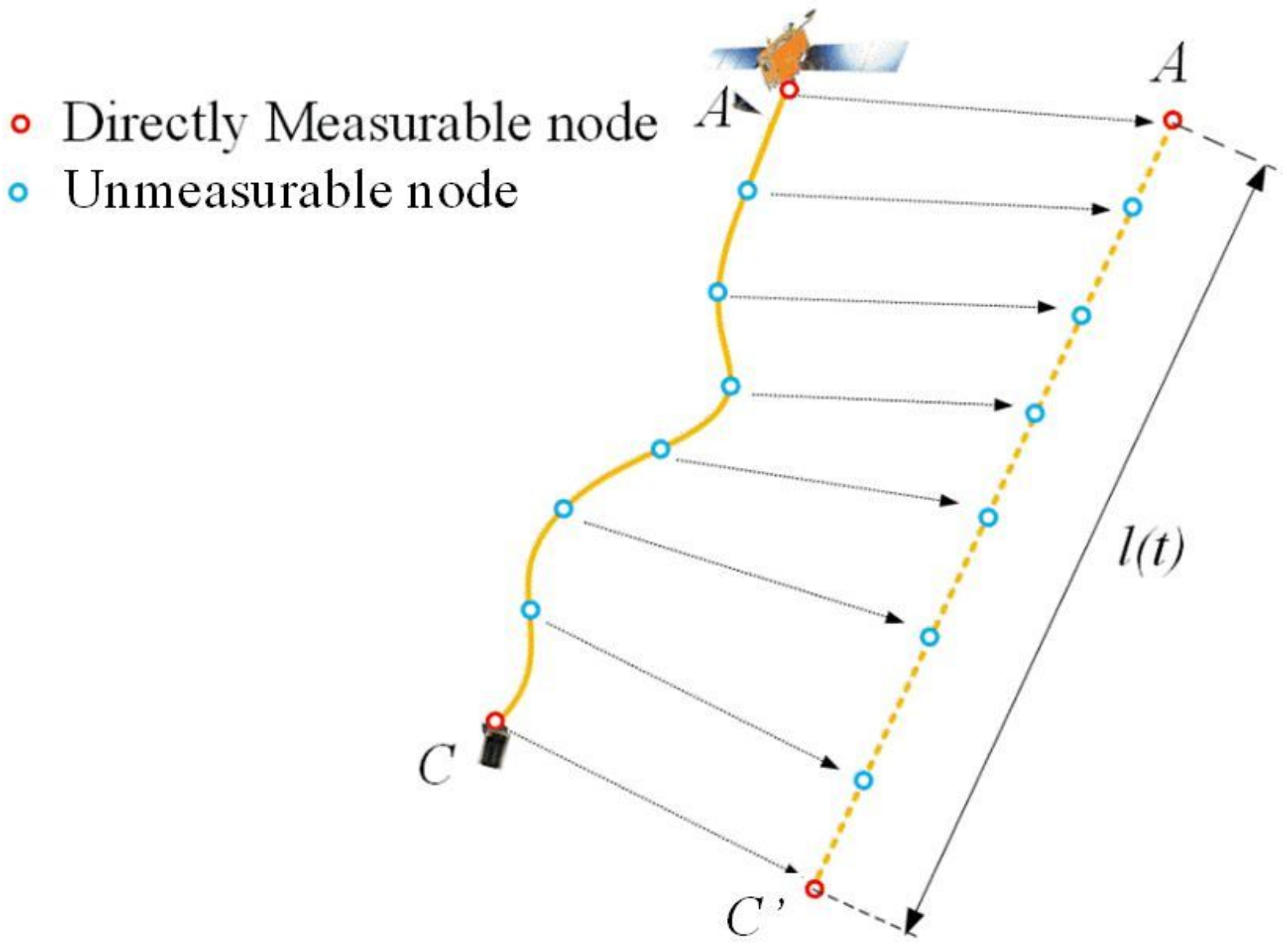

Figure 3

Schematic diagram for generation of reference tether. 


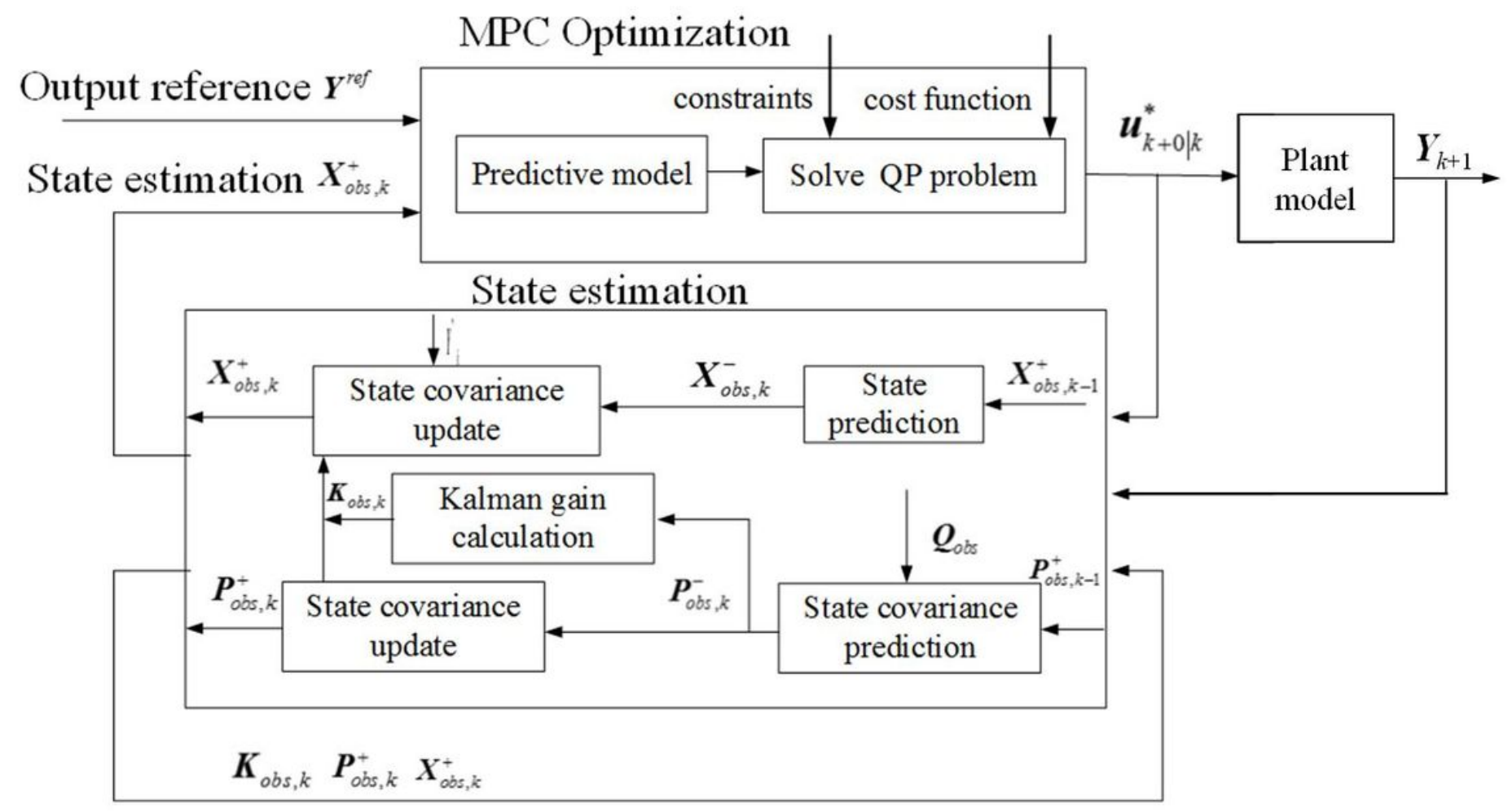

Figure 4

Block diagram of the proposed MPC system with FEKF estimator. 


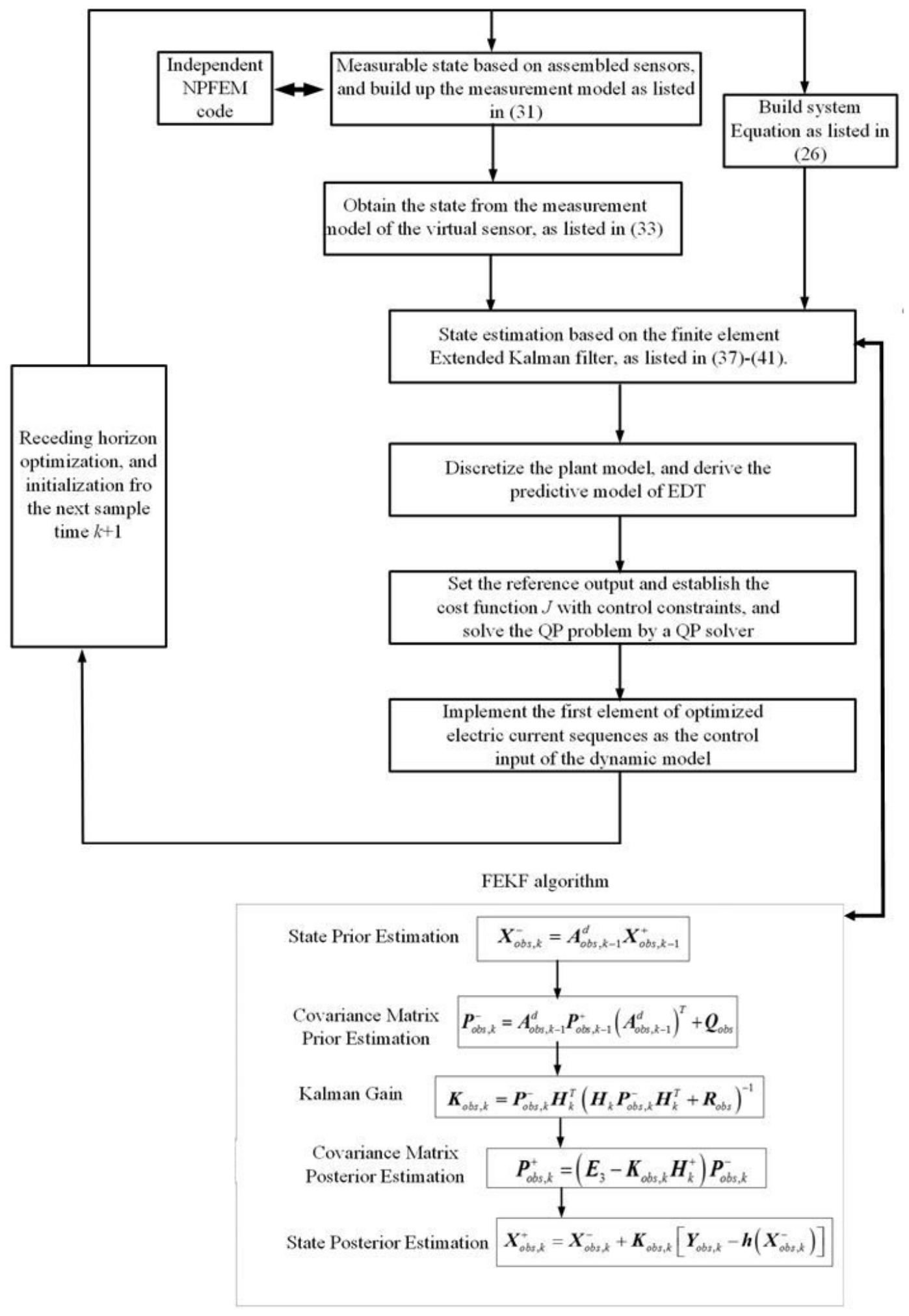

Figure 5

Flowchart of implementation of MPC with FEKF estimator. 


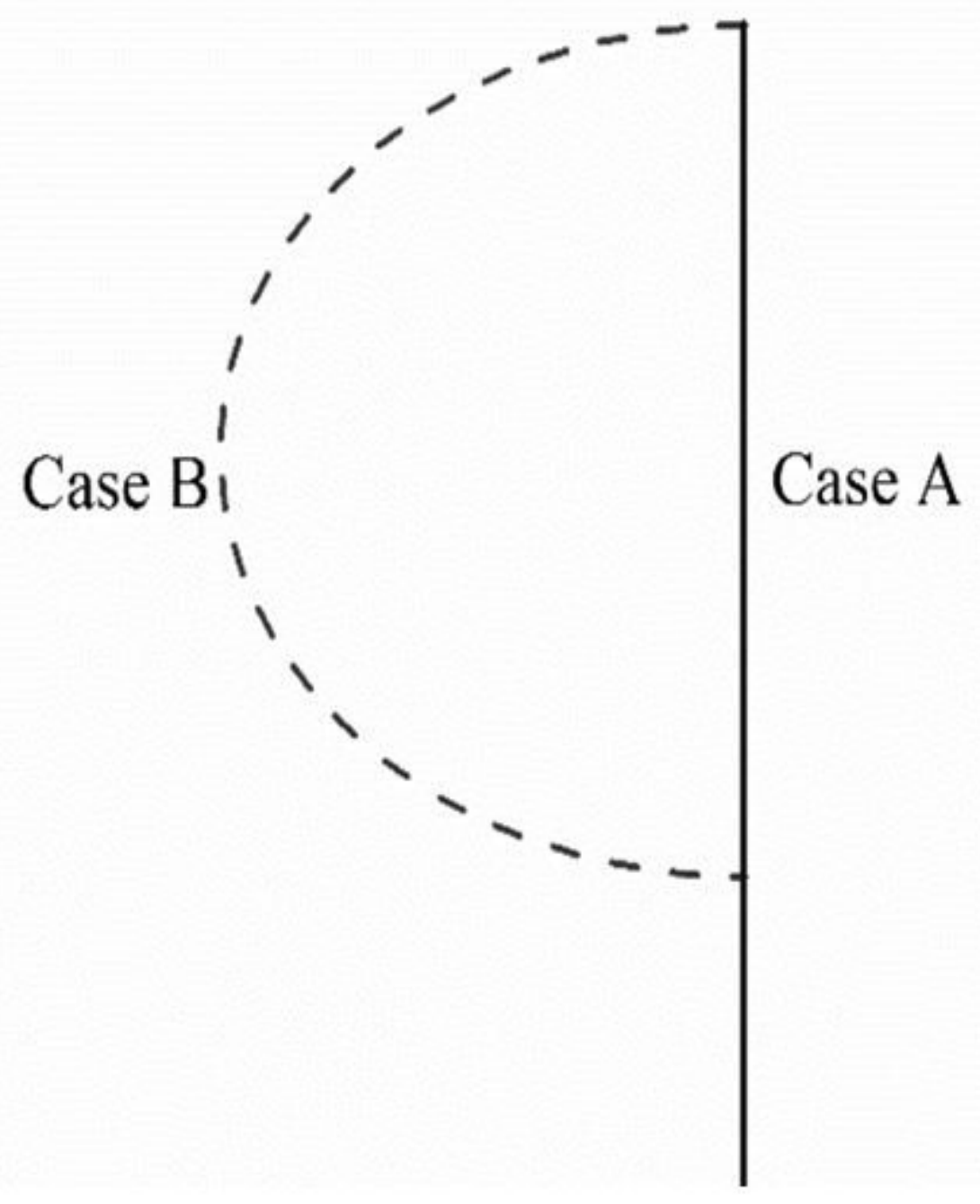

Figure 6

Tether profile of an EDT of cases A and B. 

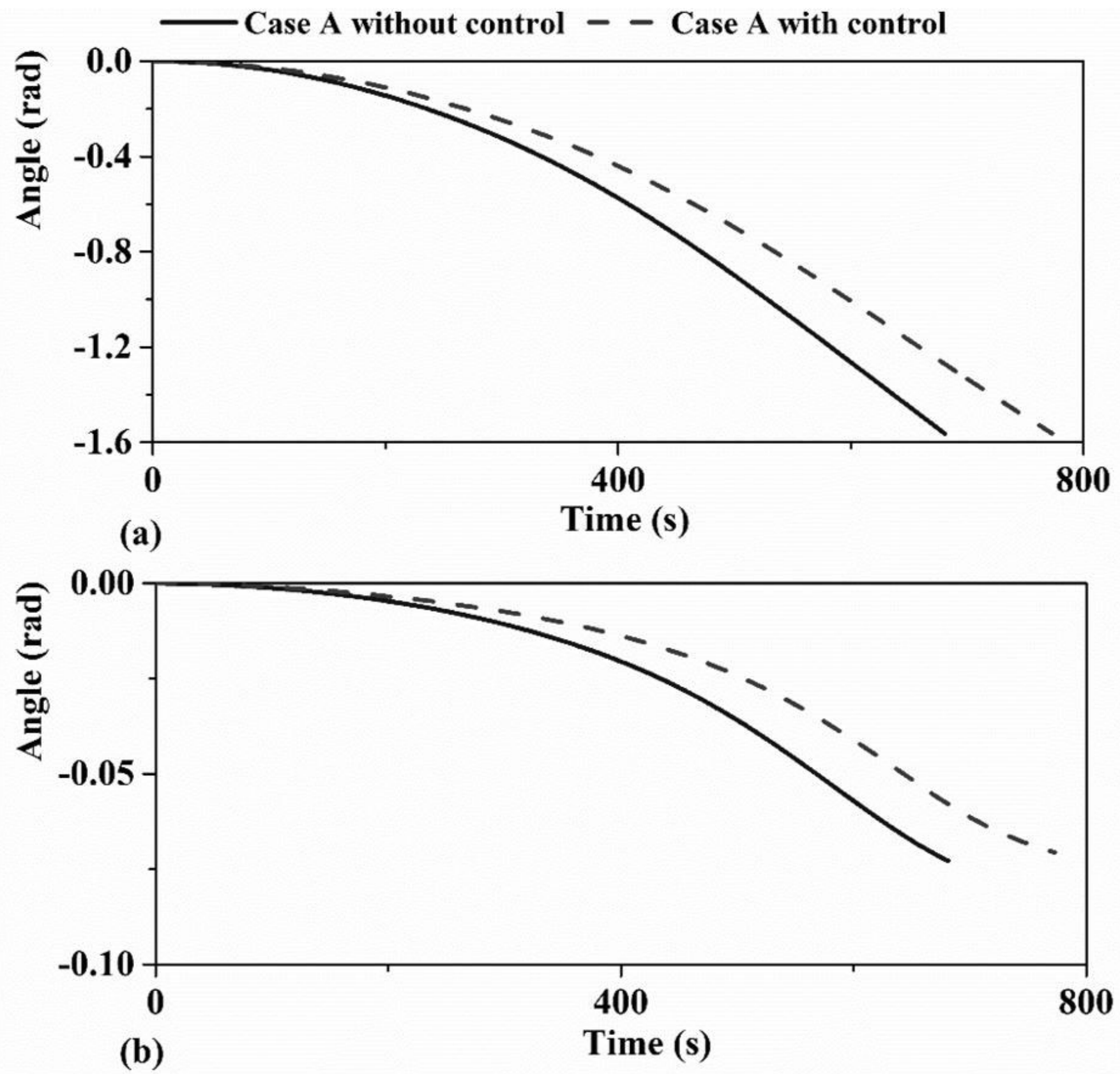

Figure 7

Libration angles of EDT at case A (a) in-plane, (b) out-of-plane. 
- Case A without controller $\rightarrow$ Case A with controller
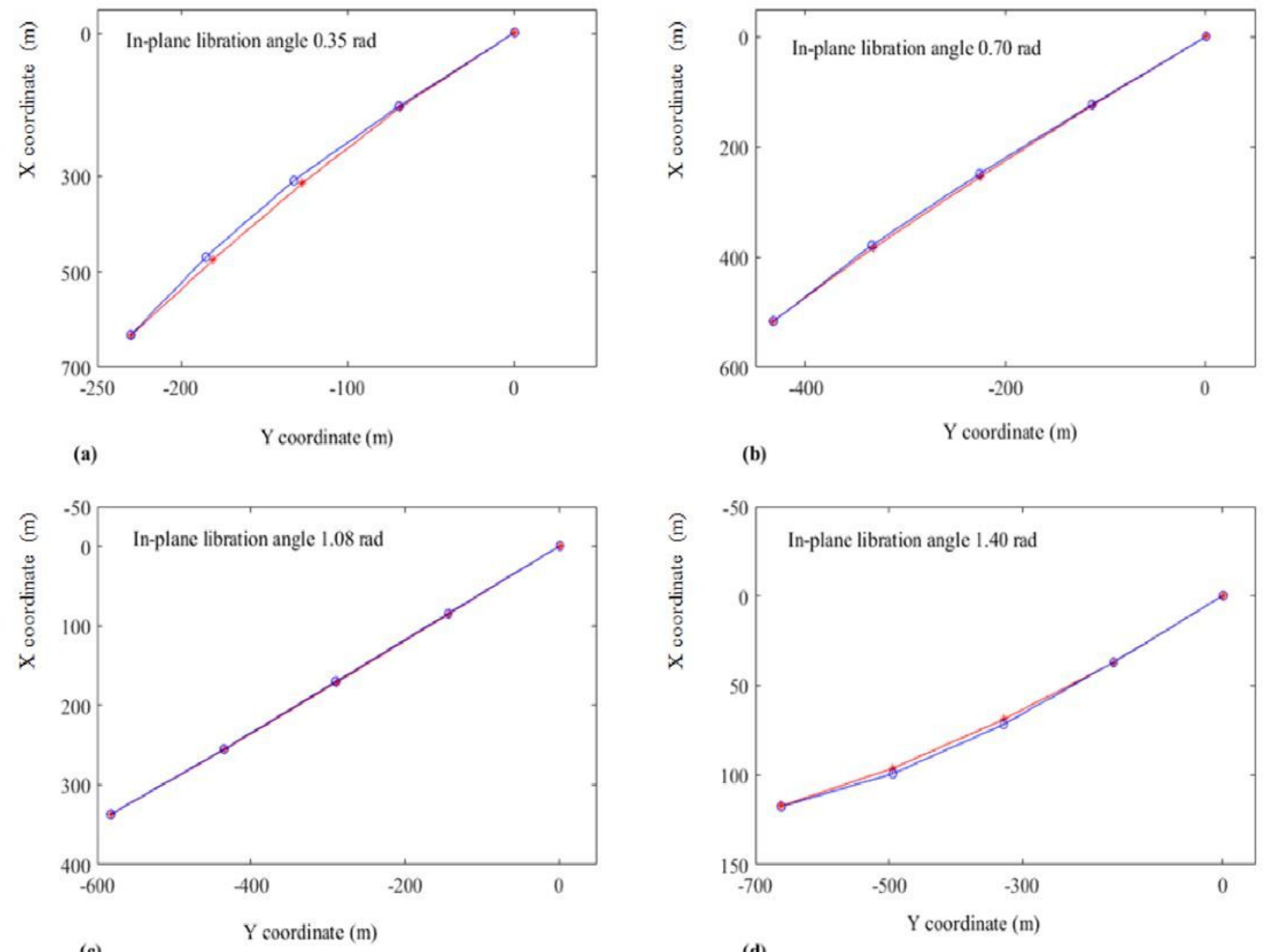

\section{Figure 8}

Geometric configuration of the flexible EDT at case A. 

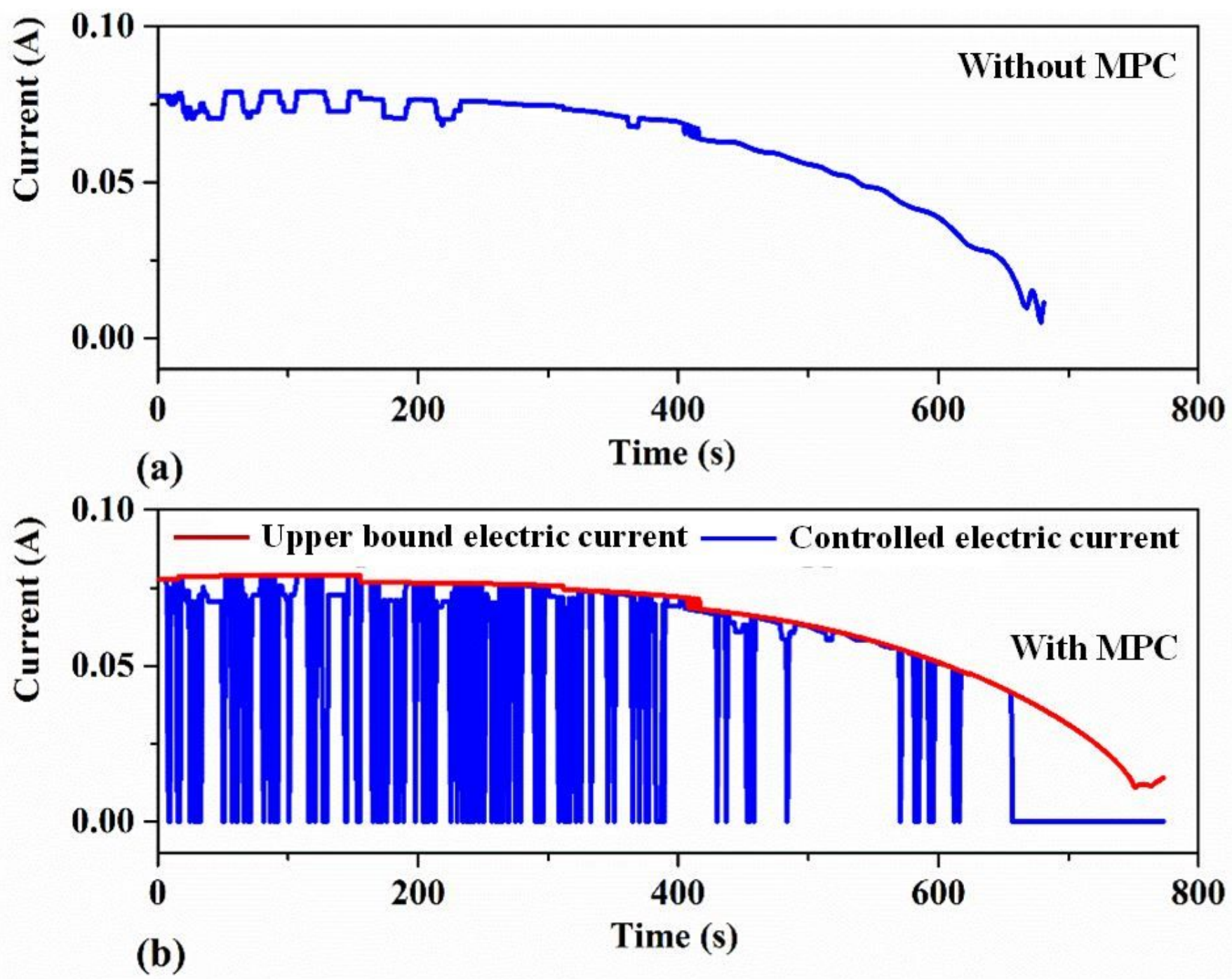

Figure 9

Case A: Electric current at cathodic end. 
- Case B without controller - - Case B with controller
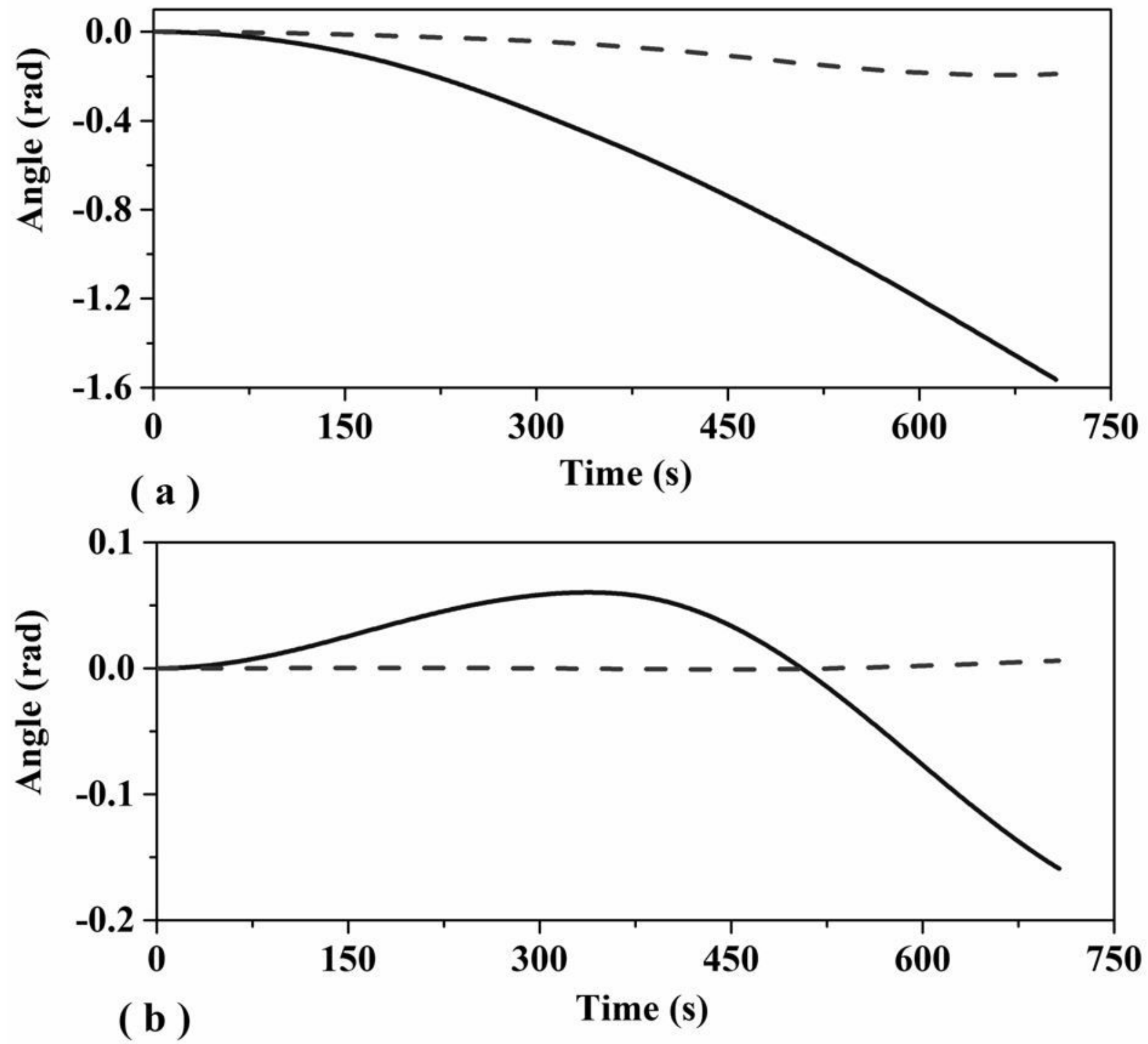

Figure 10

Libration angle of EDT at case B (a) in-plane, (b) out-of-plane. 
- Case B without controller $\longrightarrow$ Case B with controller
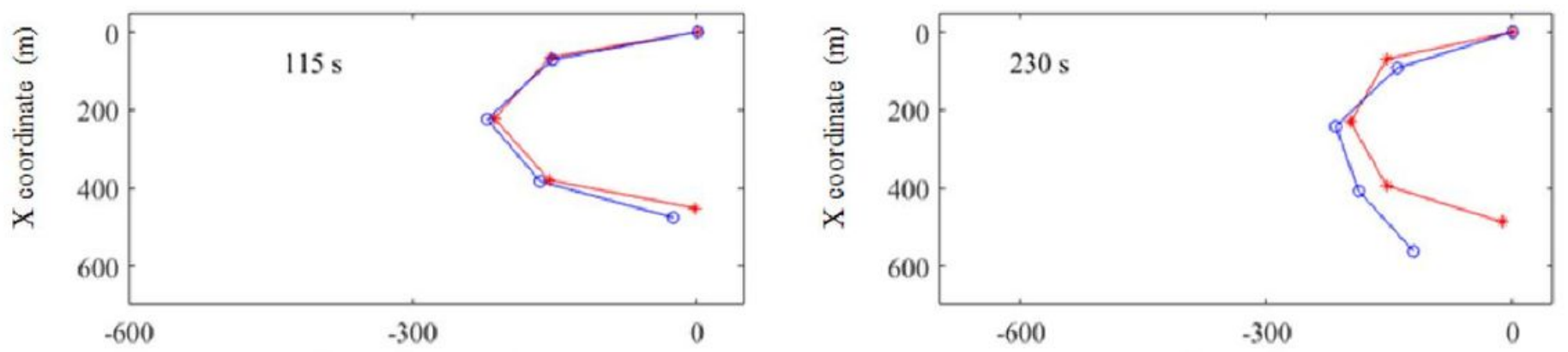

(a) Y coordinate (m)

(b)

$\mathrm{Y}$ coordinate $(\mathrm{m})$
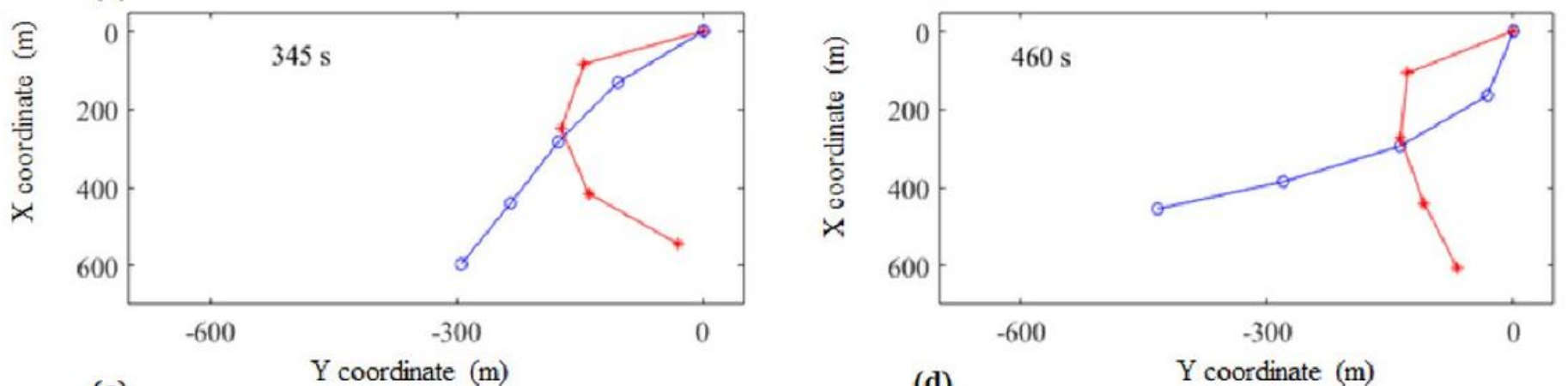

(c)

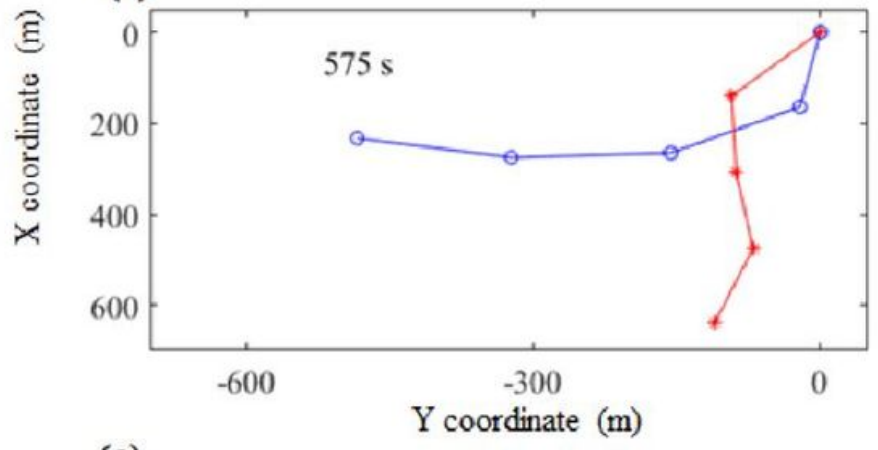

(e)

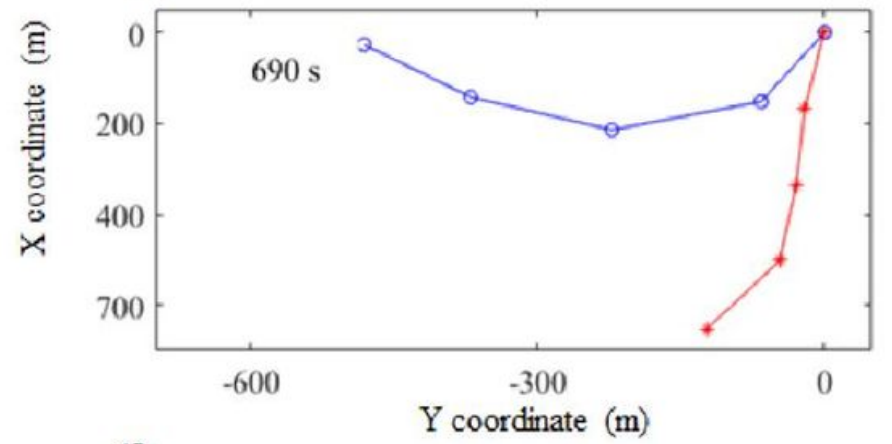

(f)

Figure 11

Geometric configuration of the flexible EDT at case B. 

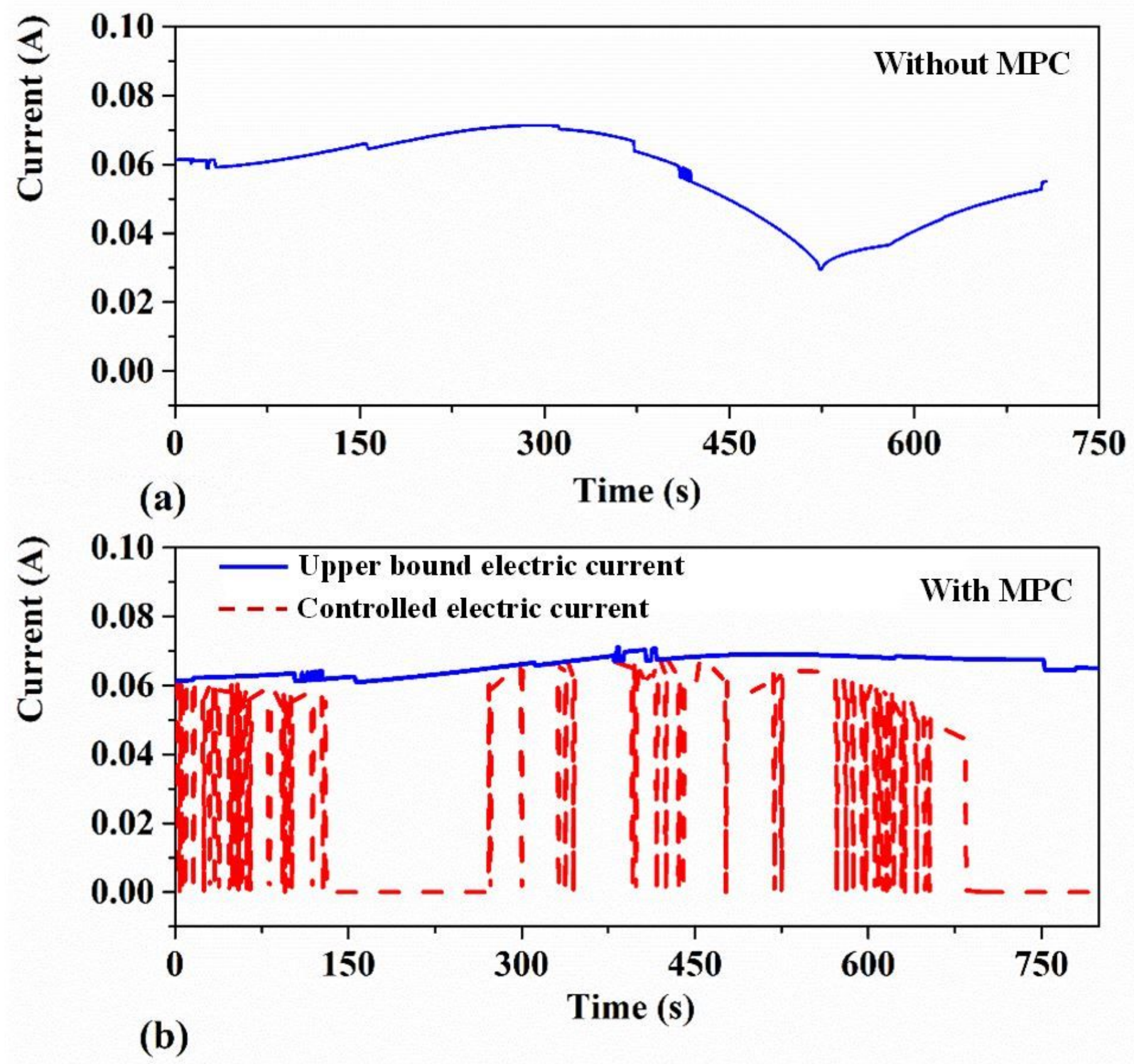

Figure 12

Case B: Electric current at cathodic end. 